\title{
A General Formula for the Mismatch Capacity
}

\author{
Anelia Somekh-Baruch
}

\begin{abstract}
The fundamental limits of channels with mismatched decoding are addressed. A general formula is established for the mismatch capacity of a general channel, defined as a sequence of conditional distributions with a general decoding metrics sequence. We deduce an identity between the Verdú-Han general channel capacity formula, and the mismatch capacity formula applied to Maximum Likelihood decoding metric. Further, several upper bounds on the capacity are provided, and a simpler expression for a lower bound is derived for the case of a non-negative decoding metric. The general formula is specialized to the case of finite input and output alphabet channels with a type-dependent metric. The closely related problem of threshold mismatched decoding is also studied, and a general expression for the threshold mismatch capacity is obtained. As an example of threshold mismatch capacity, we state a general expression for the erasures-only capacity of the finite input and output alphabet channel. We observe that for every channel there exists a (matched) threshold decoder which is capacity achieving. Additionally, necessary and sufficient conditions are stated for a channel to have a strong converse. Csiszár and Narayan's conjecture is proved for bounded metrics, providing a positive answer to the open problem introduced in [1], i.e., that the "product-space" improvement of the lower random coding bound, $C_{q}^{(\infty)}(W)$, is indeed the mismatch capacity of the discrete memoryless channel $W$. We conclude by presenting an identity between the threshold capacity and $C_{q}^{(\infty)}(W)$ in the DMC case.
\end{abstract}

A. Somekh-Baruch is with the Faculty of Engineering at Bar-Ilan University, Ramat-Gan, Israel. Email: somekha@biu.ac.il. This paper was submitted to the IEEE Transactions on Information Theory. 


\section{INTRODUCTION}

Maximum likelihood (ML) decoding is the decoding rule which minimizes the average error probability in deciding among several hypotheses. In certain setups of channel coding, due to practical limitations such as errors in channel estimation or limited resources, the decoder has a fixed structure which does not match the actual channel over which information is transmitted. This setup is referred to as mismatched decoding. Mismatched decoding has been studied extensively, especially for discrete memoryless channels (DMCs). It is usually assumed that the decoding rule maximizes, among all the codewords, a certain accumulated metric between the channel output sequence and the codeword. The highest achievable rate using a given decoder is referred to as the mismatch capacity which is obtained by optimizing over the set of possible encoding strategies.

Achievable rates for the discrete memoryless mismatched channel using random coding were derived by Csiszár and Körner [2] and by Hui [3]. Lapidoth [4] introduced an improved lower bound on the mismatch capacity of the DMC by studying the achievable sum-rate of an appropriately chosen mismatched MAC, whose codebook is obtained by expurgating codewords from the product of the codebooks of the two users. In [5], [6] the achievable region and error exponents of a cognitive MAC were considered, using superposition coding or random binning, whose sum-rate serves as a lower bound on the capacity of the single user channel. An improved bound was presented by Scarlett et al. using a refinement of the superposition coding ensemble. For given auxiliary random variables, the results of [5]-[7] may yield improved achievable rates for the DMC. In [2], an error exponent for random coding with fixed composition codes and mismatched decoding was established using a graph decomposition theorem. For other related works and extensions see [8]-[18] and references therein.

Upper bounds on the mismatch capacity have received much less attention relatively to the lower bounds. Except for some special channels, the best known upper bound on the mismatch capacity is the capacity of the same channel with matched ML decoding. A converse theorem for the mismatched binary-input DMC was proved in [19], but in general, the problem of determining the mismatch capacity of the DMC or providing a non-trivial upper bound on it, remains open.

In [1], the mismatch capacity of the DMC with decoding metric $q$, denoted $C_{q}(W)$, is considered. It is shown that the lower bound derived previously by Csiszár and Körner [2] and by Hui [3] is not tight in general but its positivity is a necessary condition for positive mismatch capacity. This result is established by proving that the random coding bound for the product channel $W_{Y_{1}, \ldots, Y_{K} \mid X_{1}, \ldots, X_{K}}=\prod_{i=1}^{K} W_{Y_{i} \mid X_{i}}$ (K consecutive channel uses of the DMC $W$ ), denoted $C_{q}^{(K)}(W)$, may result in strictly higher achievable rates. They refer to the improved bound as the "product-space" improvement of the lower bound, and the supremum of the achievable rates obtained by taking the limit of $C_{q}^{(K)}(W)$ as $K$ tends to infinity is denoted $C_{q}^{(\infty)}(W)$. In the special case of erasures-only (e.o.) capacity, the product space improvement is shown to be tight, but the question of whether this bound is tight in general remains open, and it is conjectured to be tight. It is further stated in [1] that "although the bound is not computable, its tightness would afford some valuable conclusions, for instance, that for $R<C_{q}(W)$, codes with $d$-decoding always exist with rates approaching $R$ and probability of error approaching zero exponentially fast." 
Another implication of an affirmative answer to the conjecture concerns the threshold capacity of the DMC. The threshold capacity is the supremum of achievable rates obtained by decoding the unique message which accumulates a metric that exceeds a predetermined threshold. It is stated in [1] that if the conjecture is true, then the threshold capacity and the mismatch capacity of the DMC are equal.

In this paper, the problem of mismatched decoding is addressed. A general formula for the mismatch capacity of a general channel, defined as a sequence of conditional distributions with a general decoding metrics sequence is established. We present two proofs for the upper bound on the mismatch capacity. The general capacity formula yields an identity between the Verdú-Han channel capacity formula, and the mismatch capacity formula applied to Maximum Likelihood decoding metric. Since the general capacity formula is not computable, we further provide two upper bounds on the capacity in terms of supremum over input processes of the infimum over a class of channels of the resulting spectral inf-mutual information rates. We also derive a simpler lower bound expression for the case of a non-negative decoding metric, including the special case of Mismatched Maximum Likelihood (MML) decoder, which is tailored for a channel which is different from the one over which transmission occurs. Further, the general formula is specialized to the case of finite input and output alphabet channels with type-dependent metric. We study the closely related problem of threshold mismatched decoding, and obtain a general expression for the threshold mismatch capacity. As an example of threshold mismatch capacity, we state a general expression for the erasures-only capacity of the finite input and output alphabet channel. We observe that for every channel there exists a (matched) threshold decoder which is capacity achieving. We further provide necessary and sufficient conditions for a channel to have a strong converse. Although the obtained expression of the general capacity formula is given in terms of a limiting expression which is not computable, it enables to prove Csiszár and Narayan's conjecture, hence providing a positive answer to the Open Problem 6 introduced in [1], i.e., that $C_{q}^{(\infty)}(W)$ is indeed the mismatch capacity of the DMC $W$.

The affirmative answer to Csiszár and Narayan's conjecture results in an affirmative answer to the open problems 5 and 7 raised in [1], i.e., it can be concluded that:

- There exist codes with rates approaching the mismatch capacity and probability of error decaying to zero exponentially fast as the block length goes to $\infty$.

- The threshold $d$-capacity of a DMC is equal to its mismatch $d$-capacity, at least when the metric $d$ is bounded.

The outline of this paper is as follows. Section $\amalg$ presents notation conventions. Section statement of the problem and definitions. In Section IV] a general formula for the mismatch capacity is derived, a lower bound on the capacity for non-negative mismatched metric is derived, and two alternative upper bounds on the mismatch capacity are presented. The threshold capacity is addressed in Section $\nabla$ The mismatch capacity of the DMC, as well as related special cases, are studied in section VI In Section VII, we analyze the random coding over a given codebook which result in an additional proof of the converse part of the coding theorem for the general formula of the mismatch capacity. Section VIII presents conditions for existence of a strong converse. Finally, Section $\amalg \mathrm{X}$ develops the concluding remarks. 


\section{NOTATION}

Throughout this paper, scalar random variables are denoted by capital letters, their sample values are denoted by the respective lower case letters, and their alphabets are denoted by their respective calligraphic letters, e.g. $X$, $x$, and $\mathcal{X}$, respectively. A similar convention applies to random vectors of dimension $n$ and their sample values, which are either denoted with the same symbols in the boldface font, e.g., $\boldsymbol{x}=\left(x_{1}, \ldots x_{n}\right)$ or superscripted by $n$, i.e., $x^{n}$. The set of all $n$-vectors with components taking values in a certain finite alphabet are denoted by the same alphabet superscripted by $n$, e.g., $\mathcal{X}^{n}$. The notation $X \sim P$ will stand for $P$ being the distribution of the random variable $X$.

Information theoretic quantities such as entropy, conditional entropy, and mutual information are denoted following the usual conventions in the information theory literature, e.g., $H(X), H(X \mid Y), I(X ; Y)$ and so on. To emphasize the dependence of the quantity on a certain underlying probability distribution, say $\mu$, it is subscripted by $\mu$, i.e., with notations such as $H_{\mu}(X), H_{\mu}(X \mid Y), I_{\mu}(X ; Y)$, etc. The expectation operator is denoted by $\mathbb{E}\{\cdot\}$, and once again, to make the dependence on the underlying distribution $\mu$ clear, it is denoted by $\mathbb{E}_{\mu}\{\cdot\}$. The cardinality of a finite set $A$ is denoted by $|A|$. The indicator function of an event $\mathcal{E}$ is denoted by $1\{\mathcal{E}\}$.

Let $\mathcal{P}(\mathcal{X})$ denote the set of all probability distributions on $\mathcal{X}$. For a given sequence $y \in \mathcal{Y}^{n}, \mathcal{Y}$ being a finite alphabet, $\hat{P}_{\boldsymbol{y}}$ denotes the empirical distribution on $\mathcal{Y}$ extracted from $\boldsymbol{y}$, in other words, $\hat{P}_{\boldsymbol{y}}$ is the vector $\{\hat{P} \boldsymbol{y}(y), y \in \mathcal{Y}\}$, where $\hat{P} \boldsymbol{y}(y)$ is the relative frequency of the symbol $y$ in the vector $\boldsymbol{y}$. The type-class of $\boldsymbol{x}$ is the set of $\boldsymbol{x}^{\prime} \in \mathcal{X}^{n}$ such that $\hat{P}_{\boldsymbol{x}^{\prime}}=\hat{P} \boldsymbol{x}$, which is denoted $T\left(\hat{P}_{\boldsymbol{x}}\right)$. The set of empirical distributions of order $n$ on alphabet $\mathcal{X}$ is denoted $\mathcal{P}_{n}(\mathcal{X})$.

When $\mathcal{X}$ is a finite alphabet we take a particular interest in the subset of $\mathcal{P}\left(\mathcal{X}^{n}\right)$, denoted $\mathcal{P}_{C C}(\mathcal{X}, n)$, which includes the p.m.f.'s which assign positive value to sequences that lie in a certain single type-class, i.e.,

$$
\mathcal{P}_{C C}(\mathcal{X}, n)=\left\{P \in \mathcal{P}\left(\mathcal{X}^{n}\right): \exists Q \in \mathcal{P}_{n}(\mathcal{X}) \text { s.t. } P\left(x^{n}\right)=0 \forall x^{n} \notin T(Q)\right\},
$$

note that $P$ need not necessarily be uniform within that type-class, nor does it necessarily assign positive value to all members of that type-class.

For two sequences of positive numbers, $\left\{a_{n}\right\}$ and $\left\{b_{n}\right\}$, the notation $a_{n} \doteq b_{n}$ means that $\left\{a_{n}\right\}$ and $\left\{b_{n}\right\}$ are of

the same exponential order, i.e., $\frac{1}{n} \ln \frac{a_{n}}{b_{n}} \rightarrow 0$ as $n \rightarrow \infty$. Similarly, $a_{n} \leq b_{n}$ means that $\lim _{\sup _{n}} \frac{1}{n} \ln \frac{a_{n}}{b_{n}} \leq 0$, and so on. Throughout this paper logarithms are taken to base 2 .

\section{PRELIMINARIES}

In this paper, general single-user channels which are not restricted to be stationary memoryless nor ergodic are considered. We adopt the following definition of [20] of a general channel.

Definition 1. A channel $\boldsymbol{W}=W^{(n)}, n=1,2, \ldots$ is an arbitrary sequence of increasing dimension where $W^{(n)}$ is a conditional output distribution from $\mathcal{X}^{n}$ to $\mathcal{Y}^{n}$, where $\mathcal{X}$ and $\mathcal{Y}$ are the input and output alphabets, respectively.

\footnotetext{
${ }^{1}$ In fact, as in [20], the discussion can be easily extended to input alphabets which are not necessarily Cartesian products of increasing order of the same alphabet $\mathcal{X}$.
} 
With a little abuse of terminology we shall refer to $\boldsymbol{W}$ as well as to $W^{(n)}$ as channels, where the exact meaning will be clear from the context. We note that, unless stated otherwise, throughout the paper, as in [20], we assume for simplicity that the alphabets $\mathcal{X}$ and $\mathcal{Y}$ are finite or countably infinite. If either alphabet is general, proper modifications should be made such as replacing summations with integrals etc.

A rate- $R$ block-code of length $n$ consists of $2^{n R} n$-vectors $\boldsymbol{x}(m), m=1,2, \ldots, 2^{n R}$, which represent $2^{n R}$ different messages, i.e., it is defined by the encoding function

$$
f_{n}:\left\{1, \ldots, 2^{n R}\right\} \rightarrow \mathcal{X}^{n}
$$

It is assumed that all possible messages are a-priori equiprobable, i.e., $P(m)=2^{-n R}$ for all $m$, and the random message is denoted by $S$.

A mismatched decoder for the channel is defined by a mapping

$$
q_{n}: \mathcal{X}^{n} \times \mathcal{Y}^{n} \rightarrow \mathbb{R}
$$

where the decoder declares that message $i$ was transmitted iff

$$
q_{n}(\boldsymbol{x}(i), \boldsymbol{y})>q_{n}(\boldsymbol{x}(j), \boldsymbol{y}), \forall j \neq i
$$

and if no such $i$ exists, an error is declared.

Following are several useful definitions.

Definition 2. A code $\mathcal{C}_{n}$ with decoding metric $q_{n}$ is an $\left(n, M_{n}, \epsilon\right)$-code for the channel $W^{(n)}$ if it has $M_{n}$ codewords of length $n$ and the average probability of error incurred by the decoder $q_{n}$ applied to the codebook $\mathcal{C}_{n}$ and the output of the channel $W^{(n)}$ is no larger than $\epsilon$.

In certain cases, it will be useful to omit the average probability of error $\epsilon_{n}$ from the notation and to refer to a code which has $M_{n}$ codewords of length $n$ as an $\left(n, M_{n}\right)$-code, it will also be useful to define the average probability of error associated with a codebook, a channel and a metric:

Definition 3. For a given codebook $\mathcal{C}_{n}$, let $P_{e}\left(W^{(n)}, \mathcal{C}_{n}, q_{n}\right)$ designate the average probability of error incurred by the decoder $q_{n}$ employed on the output of the channel $W^{(n)}$.

We next define an $\epsilon$-achievable rate and the mismatch capacity.

Definition 4. A rate $R>0$ is an $\epsilon$-achievable rate for the channel $\boldsymbol{W}$ with decoding metrics sequence $\boldsymbol{q}=\left\{q_{n}\right\}_{n \geq 1}$ if for every $\delta>0$, there exists a sequence of codes $\left\{\mathcal{C}_{n}\right\}_{n \geq 1}$ such that for all $n$ sufficiently large, $\mathcal{C}_{n}$ is an $\left(n, M_{n}, \epsilon\right)$ code for the channel $W^{(n)}$ and decoding metric $q_{n}$ with rate $\frac{\log \left(M_{n}\right)}{n}>R-\delta$.

Definition 5. The capacity of the channel $\boldsymbol{W}=\left\{W^{(n)}\right\}_{n \geq 1}$ with decoding metrics sequence $\boldsymbol{q}=\left\{q_{n}\right\}_{n \geq 1}$ (or, the mismatch $\boldsymbol{q}$-capacity of the channel $\boldsymbol{W})$, denoted $C_{\boldsymbol{q}}(\boldsymbol{W})$, is the supremum of rates that are $\epsilon$-achievable for all $0<\epsilon<1$. 
A closely related notion to that of mismatched $q_{n}$-decoder is the $\left(q_{n}, \tau_{n}\right)$-threshold decoder which decides that $i$ is the transmitted message iff

$$
q_{n}(\boldsymbol{x}(i), \boldsymbol{y}) \geq \tau_{n}
$$

and

$$
q_{n}(\boldsymbol{x}(j), \boldsymbol{y})<\tau_{n}, \forall j \neq i
$$

We distinguish between two setups of threshold decoding.

Definition 6. The threshold $\boldsymbol{q}$-capacity of a channel $\boldsymbol{W}$, denoted $C_{\boldsymbol{q}}^{\text {thresh }}(\boldsymbol{W})$, is defined as the supremum of rates attainable by codes with $\left(q_{n}, \tau_{n}\right)$-threshold decoders of the form (5)-(6) and any threshold sequence $\tau_{n}, n \geq 1$.

Definition 7. The constant threshold $\boldsymbol{q}$-capacity of a channel, denoted $C_{\boldsymbol{q}}^{\text {const,thresh }}(\boldsymbol{W})$, is defined as the supremum of the rates attainable by codes with $\left(q_{n}, \tau\right)$-threshold decoders for a constant $\tau$ (which does not depends on $n$ ).

Clearly,

$$
C_{\boldsymbol{q}}^{\text {const,thresh }}(\boldsymbol{W}) \leq C_{\boldsymbol{q}}^{\text {thresh }}(\boldsymbol{W}) \leq C_{\boldsymbol{q}}(\boldsymbol{W}),
$$

where the last equality follow 2 since a threshold decoder (5)-6 is more restrictive than the mismatched decoder (4).

\section{A General Formula for the Mismatch CAPACity}

In this section, we derive a general formula for the mismatch capacity. The general formula holds for general sequences of decoding metrics $q_{n}$ (3).

The following notation will be useful in what follows. Let $\boldsymbol{q}$ be a given sequence $\left\{q_{i}\right\}_{i \geq 1}$ of decoding metrics. For $\mu$, a distribution of a random variable $\tilde{X}^{n}$ on $\mathcal{X}^{n}$, a real number $c$, and an $n$-vector $\boldsymbol{y} \in \mathcal{Y}^{n}$, define the following function

$$
\begin{aligned}
\Phi_{q_{n}}(c, \mu, \boldsymbol{y}) & =\mathbb{E}_{\mu}\left(1\left\{q_{n}\left(\tilde{X}^{n}, \boldsymbol{y}\right) \geq c\right\}\right) \\
& =\mu\left\{q_{n}\left(\tilde{X}^{n}, \boldsymbol{y}\right) \geq c\right\} \\
& =\sum_{\tilde{\boldsymbol{x}} \in \mathcal{X}^{n}: q_{n}(\tilde{\boldsymbol{x}}, \boldsymbol{y}) \geq c} \mu(\tilde{\boldsymbol{x}}) .
\end{aligned}
$$

Although general alphabets are not treated in this paper, we note that in the case in which $\mu$ is a distribution on a general alphabet $\mathcal{X}^{n}$, one has

$$
\Phi_{q_{n}}(c, \mu, \boldsymbol{y}) \triangleq \int_{\tilde{\boldsymbol{x}}: q_{n}(\tilde{\boldsymbol{x}}, \boldsymbol{y}) \geq c} d \mu(\tilde{\boldsymbol{x}}),
$$

where the notation $\int$ refers to Lebesgue integral.

\footnotetext{
${ }^{2}$ For a formal proof of this claim see Lemma 3
} 
Another term that will be used throughout the paper is the limit inferior in probability of a sequence of random variables.

Definition 8. [20] The limit inferior in probability of a sequence of random variables $X_{n}, n \geq 1$, denoted p-limsup $X_{n}$, is the supremum of all $\alpha \in \mathbb{R}$ such tha $3 \lim _{\sup _{n \rightarrow \infty}} \operatorname{Pr}\left\{X_{n}<\alpha\right\}=0$, i.e.,

$$
p \text {-liminf } X_{n}=\sup \left\{\alpha: \limsup _{n \rightarrow \infty} \operatorname{Pr}\left\{X_{n}<\alpha\right\}=0\right\}
$$

We note that we adopt the definition of [21] with strict inequality $\left\{X_{n}<\alpha\right\}$ rather than that of [20] with loose inequality $\left\{X_{n} \leq \alpha\right\}$. The reason for this choice is explained in the sequel (see (49)-(50)).

Definition 9. The limit superior in probability of a sequence of random variables $X_{n}, n \geq 1$, denoted $p$-limsup $X_{n}$, is the infimum of all $\beta \in \mathbb{R}$ such that $\lim _{\sup _{n \rightarrow \infty}} \operatorname{Pr}\left\{X_{n}>\alpha\right\}=0$, i.e.,

$$
\text { p-limsup } X_{n}=\inf \left\{\beta: \limsup _{n \rightarrow \infty} \operatorname{Pr}\left\{X_{n}>\beta\right\}=0\right\} \text {. }
$$

Define the set of sequences of distributions of increasing dimension

$$
\mathcal{P}^{(\infty)} \triangleq\left\{\boldsymbol{P}=\left\{P^{(n)}\right\}_{n \geq 1}: \forall n, P^{(n)} \in \mathcal{P}\left(\mathcal{X}^{n}\right)\right\}
$$

Define the subset of $\mathcal{P}^{(\infty)}$ containing sequences of distributions which are uniform over their support, i.e.,

$$
\mathcal{P}_{U}^{(\infty)} \triangleq\left\{\boldsymbol{P}=\left\{P^{(n)}\right\}_{n \geq 1} \in \mathcal{P}^{(\infty)}: \forall n, P^{(n)}\left(\tilde{x}^{n}\right)=P^{(n)}\left(x^{n}\right) \text { if } P^{(n)}\left(x^{n}\right) \cdot P^{(n)}\left(\tilde{x}^{n}\right)>0\right\}
$$

For a sequence of distributions $\boldsymbol{P}=\left\{P^{(n)}\right\}_{n \geq 1} \in \mathcal{P}^{(\infty)}$, a channel $\boldsymbol{W}=\left\{W^{(i)}\right\}_{i \geq 1}$, and a sequence of metrics $\boldsymbol{q}=\left\{q_{i}\right\}_{i \geq 1}$, let $\left(X^{n}, Y^{n}\right) \sim P^{(n)} \times W^{(n)}$ and denote

$$
\underline{K}_{\boldsymbol{q}}(\boldsymbol{P}, \boldsymbol{W}) \triangleq p-\liminf -\frac{1}{n} \log \left(\Phi_{q_{n}}\left(q_{n}\left(X^{n}, Y^{n}\right), P^{(n)}, Y^{n}\right)\right)
$$

The multi-letter expression for the mismatch capacity is stated in the following theorem.

Theorem 1. The mismatch $\boldsymbol{q}$-capacity of the channel $\boldsymbol{W}$ is given by

$$
C_{\boldsymbol{q}}(\boldsymbol{W})=\sup _{\boldsymbol{P} \in \mathcal{P}(\infty)} \underline{K}_{\boldsymbol{q}}(\boldsymbol{P}, \boldsymbol{W})
$$

where the supremum can be restricted to $\boldsymbol{P} \in \mathcal{P}_{U}^{(\infty)}$.

Before we prove the theorem, a few comments are in order.

- Observe that if $X^{n}$ and $Y^{n}$ designate the channel input and output, respectively, and $P^{(n)}$ is uniform over an $\left(n, M_{n}\right)$ codebook, $\Phi_{q_{n}}\left(q_{n}\left(X^{n}, Y^{n}\right), P^{(n)}, Y^{n}\right)$ can be regarded as the conditional error probability given $\left(X^{n}, Y^{n}\right)$ in a single drawing of another codeword $\tilde{X}^{n}$ uniformly over the codebook. Hence, the capacity formula is the supremum over input distribution sequence of the limit inferior in probability of the exponent

${ }^{3}$ Unlike the definition in [20], it is required that $\limsup _{n \rightarrow \infty} \operatorname{Pr}\left\{X_{n}<\alpha\right\}=0$ rather than $\lim _{n \rightarrow \infty} \operatorname{Pr}\left\{X_{n}<\alpha\right\}=0$ since in certain cases, the sequence $a_{n}=\operatorname{Pr}\left\{X_{n}<\alpha\right\}$ might not converge to a limit. 
of the conditional error probability in a single drawing of another codeword $\tilde{X}^{n}$ uniformly over the codebook. More generally, for $\mu \in \mathcal{P}\left(\mathcal{X}^{n}\right)$, and a triple of random variables

$$
\left(X^{n}, \tilde{X}^{n}, Y^{n}\right) \sim \mu\left(X^{n}\right) \mu\left(\tilde{X}^{n}\right) W^{(n)}\left(Y^{n} \mid X^{n}\right)
$$

we have

$$
\Phi_{q_{n}}\left(q_{n}\left(X^{n}, Y^{n}\right), P^{(n)}, Y^{n}\right)=\operatorname{Pr}\left\{q_{n}\left(\tilde{X}^{n}, Y^{n}\right) \geq q_{n}\left(X^{n}, Y^{n}\right) \mid X^{n}, Y^{n}\right\} .
$$

- We note that a general metric can account for any decoder with disjoint decision regions. To realize this, note that a general decoder with disjoint decision regions $\mathcal{D}_{m}, m=1, . ., M_{n}$ applied to the codebook $\mathcal{C}=\left\{\boldsymbol{x}_{m}\right\}_{m=1}^{M_{n}}$ can be expressed as a decoder with respect to the metric

$$
q_{n}\left(\boldsymbol{x}_{m}, \boldsymbol{y}\right)=1\left\{\boldsymbol{y} \in \mathcal{D}_{m}\right\}, \forall \boldsymbol{x}_{m} \in \mathcal{C} .
$$

- We also note that the proof of Theorem 1 can be extended quite straightforwardly to rather general alphabets $\mathcal{X}, \mathcal{Y}$ and appropriate $\sigma$-algebras, as long as for any probability distribution $P_{X^{n}} \in \mathcal{P}\left(\mathcal{X}^{n}\right)$, the probability distribution $P_{X^{n}} \times W_{Y^{n} \mid X^{n}}$ is well defined. In the general case, the definition of $\Phi$ should be replaced with the Lebesgue integral 9 .

Next, Theorem 1 is proved. As mentioned before, an additional proof of the converse part of the theorem is provided in Section VII.

Proof: We begin with the proof of the converse part. The following lemma implies that the highest achievable rate is upper bounded by $\sup _{\boldsymbol{P}} \underline{K}_{\boldsymbol{q}}(\boldsymbol{P}, \boldsymbol{W})$.

Lemma 1. Let $X^{n}$ be the random variable uniformly distributed over an $\left(n, M_{n}\right)$-code $\mathcal{C}_{n}$, and $Y^{n}$ the output of a channel $W^{(n)}$ with $X^{n}$ as the input, then

$$
\operatorname{Pr}\left\{-\frac{1}{n} \log \left(\Phi_{q_{n}}\left(q_{n}\left(X^{n}, Y^{n}\right), P^{(n)}, Y^{n}\right)\right)<\frac{1}{n} \log M_{n}\right\}=P_{e}\left(W^{(n)}, \mathcal{C}_{n}, q_{n}\right),
$$

where $P^{(n)}$ is the distribution of the codeword $X^{n}$, i.e., a uniform distribution over $\mathcal{C}_{n}$.

Proof: Note that

$$
\begin{aligned}
& \Phi_{q_{n}}\left(q_{n}\left(X^{n}, Y^{n}\right), P^{(n)}, Y^{n}\right) \\
= & \sum_{\boldsymbol{x}^{\prime} \in \mathcal{C}_{n}: q_{n}\left(\boldsymbol{x}^{\prime}, Y^{n}\right) \geq q_{n}\left(X^{n}, Y^{n}\right)} P^{(n)}\left(\boldsymbol{x}^{\prime}\right) \\
= & \frac{\left|\left\{\boldsymbol{x}^{\prime} \in \mathcal{C}_{n}: q_{n}\left(\boldsymbol{x}^{\prime}, Y^{n}\right) \geq q_{n}\left(X^{n}, Y^{n}\right)\right\}\right|}{M_{n}}
\end{aligned}
$$


where the last equality follows since $X^{n}$ is distributed uniformly over the codebook of size $M_{n}$. Hence, the left hand side of 19 is equal to

$$
\begin{aligned}
& \operatorname{Pr}\left\{-\frac{1}{n} \log \left(\left|\left\{\boldsymbol{x}^{\prime} \in \mathcal{C}_{n}: q_{n}\left(\boldsymbol{x}^{\prime}, Y^{n}\right) \geq q_{n}\left(X^{n}, Y^{n}\right)\right\}\right|\right)<0\right\} \\
= & \operatorname{Pr}\left\{\left|\left\{\boldsymbol{x}^{\prime} \in \mathcal{C}_{n}: q_{n}\left(\boldsymbol{x}^{\prime}, Y^{n}\right) \geq q_{n}\left(X^{n}, Y^{n}\right)\right\}\right|>1\right\} \\
= & P_{e}\left(W^{(n)}, \mathcal{C}_{n}, q_{n}\right),
\end{aligned}
$$

where the last step follows since the decision rule (4) can be rewritten as: decide $m$ iff

$$
\left|\left\{\boldsymbol{x}^{\prime} \in \mathcal{C}_{n}: q_{n}\left(\boldsymbol{x}^{\prime}, \boldsymbol{y}\right) \geq q_{n}\left(\boldsymbol{x}_{m}, \boldsymbol{y}\right)\right\}\right|=1
$$

and the corresponding decision region $\mathcal{D}_{m}$ is

$$
\mathcal{D}_{m}=\left\{\boldsymbol{y}:\left|\left\{\boldsymbol{x}^{\prime} \in \mathcal{C}_{n}: q_{n}\left(\boldsymbol{x}^{\prime}, \boldsymbol{y}\right) \geq q_{n}\left(\boldsymbol{x}_{m}, \boldsymbol{y}\right)\right\}\right|=1\right\} .
$$

This concludes the proof of Lemma 1

Now, fix $\gamma>0$ and assume in negation that $R=\sup _{\boldsymbol{P}^{\prime}} \underline{K}_{\boldsymbol{q}}\left(\boldsymbol{P}^{\prime}, \boldsymbol{W}\right)+2 \gamma$ is an achievable rate, therefore, there exists a sequence of $\left(n, M_{n}\right)$-codes, $\left\{\mathcal{C}_{n}\right\}_{n \geq 1}$, satisfying $\limsup _{n \rightarrow \infty} P_{e}\left(W^{(n)}, \mathcal{C}_{n}, q_{n}\right)=0$ and $\liminf \operatorname{in}_{n \rightarrow \infty} \frac{1}{n} \log M_{n} \geq R>\sup _{\boldsymbol{P}} \underline{K}_{\boldsymbol{q}}(\boldsymbol{P}, \boldsymbol{W})+\gamma$. Thus, from Lemma 1 we have for sufficiently large $n$,

$$
\begin{aligned}
& P_{e}\left(W^{(n)}, \mathcal{C}_{n}, q_{n}\right) \\
= & \operatorname{Pr}\left\{-\frac{1}{n} \log \left(\Phi_{q_{n}}\left(q_{n}\left(X^{n}, Y^{n}\right), P^{(n)}, Y^{n}\right)\right)<\frac{1}{n} \log M_{n}\right\} \\
\geq & \operatorname{Pr}\left\{-\frac{1}{n} \log \left(\Phi_{q_{n}}\left(q_{n}\left(X^{n}, Y^{n}\right), P^{(n)}, Y^{n}\right)\right)<\sup _{\boldsymbol{P}^{\prime}} \underline{K}_{\boldsymbol{q}}\left(\boldsymbol{P}^{\prime}, \boldsymbol{W}\right)+\gamma\right\} \\
\geq & \operatorname{Pr}\left\{-\frac{1}{n} \log \left(\Phi_{q_{n}}\left(q_{n}\left(X^{n}, Y^{n}\right), P^{(n)}, Y^{n}\right)\right)<\underline{K}_{\boldsymbol{q}}(\boldsymbol{P}, \boldsymbol{W})+\gamma\right\},
\end{aligned}
$$

and by definition of $\underline{K}_{\boldsymbol{q}}(\boldsymbol{P}, \boldsymbol{W})$, the r.h.s. of $(25)$ is bounded away from zero for infinitely many $n$ 's, and hence $P_{e}\left(W^{(n)}, \mathcal{C}_{n}, q_{n}\right)$ cannot vanish in contradiction to the assumption. We observe that since $P^{(n)}$ is uniform over $\mathcal{C}_{n}$, the supremum in (24) can be restricted to include only sequences of distributions that are uniform over a subset of their support, and this concludes the proof of the converse part of Theorem 1

Next, the direct part of Theorem 1 is proved.

Let $\boldsymbol{P}=\left\{P^{(n)}\right\}_{n \geq 1}$ be an arbitrary sequence of distributions where $P^{(n)} \in \mathcal{P}\left(\mathcal{X}^{n}\right)$. We use random coding with $P^{(n)}$ to generate $M_{n}=2^{n R}$ independent codewords constituting the codebook where

$$
R=\underline{K}_{\boldsymbol{q}}(\boldsymbol{P}, \boldsymbol{W})-2 \gamma
$$

and $\gamma>0$ can be chosen arbitrarily small. Denote by $\mathcal{A}_{n}$ the set of pairs of $n$-vectors $(\boldsymbol{x}, \boldsymbol{y}) \in \mathcal{X}^{n} \times \mathcal{Y}^{n}$ such that $\left.2^{n(\underline{K}} \boldsymbol{q}(\boldsymbol{P}, \boldsymbol{W})-\gamma\right) \cdot \Phi_{q_{n}}\left(q_{n}(\boldsymbol{x}, \boldsymbol{y}), P^{(n)}, \boldsymbol{y}\right) \leq 1$. Note that by definition of $\underline{K}_{\boldsymbol{q}}(\boldsymbol{P}, \boldsymbol{W})$ we have $\operatorname{Pr}\left\{\mathcal{A}_{n}^{c}\right\} \rightarrow 0$ as $n$ tends to infinity. Further, the ensemble average probability of error can be computed as the probability of at 
least one "failure" in $M_{n}-1$ independent Bernoulli experiments, i.e., the average probability of error denoted $\bar{P}_{e}$ satisfies

$$
\begin{aligned}
& \bar{P}_{e}=\mathbb{E}\left[1-\left(1-\Phi_{q_{n}}\left(q_{n}\left(X^{n}, Y^{n}\right), P^{(n)}, Y^{n}\right)\right)^{M_{n}-1}\right] \\
& \stackrel{(a)}{\leq} \mathbb{E} \min \left\{1, M_{n} \Phi_{q_{n}}\left(q_{n}\left(X^{n}, Y^{n}\right), P^{(n)}, Y^{n}\right)\right\} \\
& \leq \mathbb{E}\left(1\left\{\left(X^{n}, Y^{n}\right) \in \mathcal{A}_{n}\right\} \min \left\{1, M_{n} \Phi_{q_{n}}\left(q_{n}\left(X^{n}, Y^{n}\right), P^{(n)}, Y^{n}\right)\right\}\right) \\
& +\operatorname{Pr}\left\{\mathcal{A}_{n}^{c}\right\}
\end{aligned}
$$

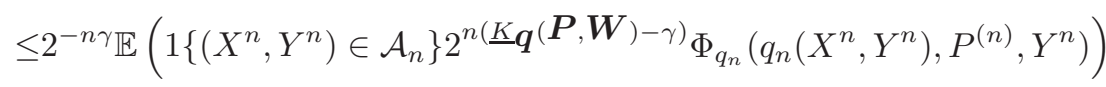

$$
\begin{aligned}
& +\operatorname{Pr}\left\{\mathcal{A}_{n}^{c}\right\} \\
& \stackrel{(b)}{\leq} 2^{-n \gamma}+\operatorname{Pr}\left\{\mathcal{A}_{n}^{c}\right\}
\end{aligned}
$$

where $\left(X^{n}, Y^{n}\right) \sim P^{(n)} \times W^{(n)},(a)$ follows from the union bound, and (b) follows by definition of $\mathcal{A}_{n}$. Therefore, we have $\bar{P}_{e} \rightarrow 0$. The vanishing ensemble average probability of error ensures that there exists a sequence of deterministic codebooks of rate $R=\underline{K}_{\boldsymbol{q}}(\boldsymbol{P}, \boldsymbol{W})-2 \gamma$ whose average probability of error using the decoding metric sequence $\boldsymbol{q}$ vanishes. The capacity formula follows since $\boldsymbol{P}$ is arbitrary, $\gamma$ can be made arbitrarily small, and by definition of the capacity as the supremum of all achievable rates.

We note the following straightforward upper bound on $C_{\boldsymbol{q}}(\boldsymbol{W})=\sup _{\boldsymbol{P}} \underline{K}_{\boldsymbol{q}}(\boldsymbol{P}, \boldsymbol{W})$.

Corollary 1. The mismatch $\boldsymbol{q}$-capacity of the channel $\boldsymbol{W}$ is upper bounded as follows

$$
C_{\boldsymbol{q}}(\boldsymbol{W}) \leq \sup _{\boldsymbol{P} \in \mathcal{P}_{U}^{(\infty)}} \liminf _{n \rightarrow \infty} \frac{1}{n} \mathbb{E} \log \frac{1}{\Phi_{q_{n}}\left(q_{n}\left(X^{n}, Y^{n}\right), P^{(n)}, Y^{n}\right)} .
$$

Proof: Let $\left\{A_{n}\right\}_{n \geq 1}$ be a sequence of non-negative random variables, and denote $\underline{A} \triangleq p$ - $\liminf A_{n}$ one has for all $\epsilon>0$,

$$
\mathbb{E}\left(A_{n}\right) \geq E\left(A_{n} 1\left\{A_{n} \geq \underline{A}-\epsilon\right\}\right) \geq(\underline{A}-\epsilon) E\left(1\left\{A_{n} \geq \underline{A}-\epsilon\right\}\right) .
$$

Therefore, by definition of $\underline{A}$, for all sufficiently large $n$

$$
\mathbb{E}\left(A_{n}\right) \geq(\underline{A}-\epsilon)(1-\epsilon),
$$

and hence,

$$
\liminf _{n \rightarrow \infty} \mathbb{E}\left(A_{n}\right) \geq \underline{A}
$$

The bound (28) follows by applying the inequality (31) to $A_{n}=-\frac{1}{n} \log \Phi_{q_{n}}\left(q_{n}\left(X^{n}, Y^{n}\right), P^{(n)}, Y^{n}\right)$, which yields

$$
\underline{K}_{\boldsymbol{q}}(\boldsymbol{P}, \boldsymbol{W}) \leq \liminf _{n \rightarrow \infty} \frac{1}{n} \mathbb{E} \log \frac{1}{\left(\Phi_{q_{n}}\left(q_{n}\left(X^{n}, Y^{n}\right), P^{(n)}, Y^{n}\right)\right)},
$$

and (28) follows by taking the supremum over $\boldsymbol{P} \in \mathcal{P}_{U}^{(\infty)}$. 
We next present a lower bound on $C_{\boldsymbol{q}}(\boldsymbol{W})$ for non-negative metrics in the spirit of [22], [9]. A non-negative metric $q_{n}$ satisfies $q_{n}\left(x^{n}, y^{n}\right) \geq 0$ for all $x^{n}, y^{n}$.

For a given channel $\boldsymbol{W}$, a non-negative decoding metrics sequence $\boldsymbol{v}=\left\{v_{n}\right\}_{n \geq 1}$, and input distributions sequence $\boldsymbol{P}=\left\{P^{(i)}\right\}_{i \geq 1}$, denote

$$
\underline{\Theta}_{\boldsymbol{v}}(\boldsymbol{P}, \boldsymbol{W}) \triangleq p-\liminf \frac{1}{n} \log \frac{v_{n}\left(X^{n}, Y^{n}\right)}{\tilde{P}^{(n)}\left(Y^{n}\right)},
$$

where $\left(X^{n}, Y^{n}\right) \sim P^{(n)} \times W^{(n)}$, and $\tilde{P}^{(n)}$ is defined as

$$
\tilde{P}^{(n)}\left(y^{n}\right)=\sum_{x^{n} \in \mathcal{X}^{n}} P^{(n)}\left(x^{n}\right) v_{n}\left(x^{n}, y^{n}\right) .
$$

Theorem 2. Let $\boldsymbol{v}=\left\{v_{i}\right\}_{i \geq 1}$ be a non-negative metrics sequence. The mismatch $\boldsymbol{v}$-capacity satisfies

$$
C \boldsymbol{v}(\boldsymbol{W}) \geq \sup _{\boldsymbol{P} \in \mathcal{P}(\infty)} \underline{\Theta}_{\boldsymbol{v}}(\boldsymbol{P}, \boldsymbol{W}) .
$$

Proof: Let $P^{(n)}$ be given. Note that

$$
\begin{aligned}
& \Phi_{v_{n}}\left(v_{n}\left(X^{n}, Y^{n}\right), P^{(n)}, Y^{n}\right) \\
&= \sum_{\boldsymbol{x}^{\prime} \in \mathcal{X}^{n}: v_{n}\left(\boldsymbol{x}^{\prime}, Y^{n}\right) \geq v_{n}\left(X^{n}, Y^{n}\right)} P^{(n)}\left(\boldsymbol{x}^{\prime}\right) \\
& \stackrel{(a)}{\leq} \sum_{\boldsymbol{x}^{\prime} \in \mathcal{X}^{n}: v_{n}\left(\boldsymbol{x}^{\prime}, Y^{n}\right) \geq v_{n}\left(X^{n}, Y^{n}\right)} P^{(n)}\left(\boldsymbol{x}^{\prime}\right) \frac{v_{n}\left(\boldsymbol{x}^{\prime}, Y^{n}\right)}{v_{n}\left(X^{n}, Y^{n}\right)} \\
&= \frac{1}{v_{n}\left(X^{n}, Y^{n}\right)} \sum_{\boldsymbol{x}^{\prime} \in \mathcal{X}^{n}: v_{n}\left(\boldsymbol{x}^{\prime}, Y^{n}\right) \geq v_{n}\left(X^{n}, Y^{n}\right)} P^{(n)}\left(\boldsymbol{x}^{\prime}\right) v_{n}\left(\boldsymbol{x}^{\prime}, Y^{n}\right) \\
& \stackrel{(b)}{\leq} \frac{1}{v_{n}\left(X^{n}, Y^{n}\right)} \sum_{\boldsymbol{x}^{\prime} \in \mathcal{X}^{n}} P^{(n)}\left(\boldsymbol{x}^{\prime}\right) v_{n}\left(\boldsymbol{x}^{\prime}, Y^{n}\right) \\
&= \frac{\tilde{P}^{(n)}\left(Y^{n}\right)}{v_{n}\left(X^{n}, Y^{n}\right)},
\end{aligned}
$$

where $(a)$ and $(B)$ follow since $v_{n}$ is a non-negative metric. Therefore,

$$
-\frac{1}{n} \log \left(\Phi_{v_{n}}\left(v_{n}\left(X^{n}, Y^{n}\right), P^{(n)}, Y^{n}\right)\right) \geq \frac{1}{n} \log \frac{v_{n}\left(X^{n}, Y^{n}\right)}{\tilde{P}^{(n)}\left(Y^{n}\right)},
$$

and thus

$$
p \text { - } \liminf -\frac{1}{n} \log \left(\Phi_{v_{n}}\left(v_{n}\left(X^{n}, Y^{n}\right), P^{(n)}, Y^{n}\right)\right) \geq p \text { - } \liminf \frac{1}{n} \log \frac{v_{n}\left(X^{n}, Y^{n}\right)}{\tilde{P}^{(n)}\left(Y^{n}\right)} .
$$

Since taking the supremum over $\boldsymbol{P}$, the left hand side of (38) becomes $C \boldsymbol{v}(\boldsymbol{W})$ by Theorem 1 (35) follows.

A few comments are in order:

- We note that the result of Theorem 2 holds for the following important class of non-negative metrics.

Definition 10. We say that a non negative metric $v_{n}:\left(\mathcal{X}^{n}, \mathcal{Y}^{n}\right) \rightarrow \mathbb{R}^{+}$is a mismatched maximum likelihood (MML) metric if $v_{n}\left(x^{n}, y^{n}\right)=V^{(n)}\left(y^{n} \mid x^{n}\right), \forall\left(x^{n}, y^{n}\right) \in \mathcal{X}^{n} \times \mathcal{Y}^{n}$ where $V^{(n)}$ is a conditional distribution from $\mathcal{X}^{n}$ to $\mathcal{Y}^{n}$, i.e., an ML decoder with respect to the channel $V^{(n)}$ with equiprobable messages. 
The class of MML decoders is relevant especially for setups in which a suboptimal decoder is used due to incorrect knowledge of the channel rather than practical limitations on its structure.

We also note that the bound of Theorem 2 is tight in the matched case, and when $\frac{1}{n} \log \frac{V^{(n)}\left(Y^{n} \mid X^{n}\right)}{\tilde{P}^{(n)}\left(Y^{n}\right)}$ converges in probability to its (possibly time-varying) expectation, we obtain,

$$
\begin{aligned}
p-\liminf \frac{1}{n} \log \frac{V^{(n)}\left(Y^{n} \mid X^{n}\right)}{\tilde{P}^{(n)}\left(Y^{n}\right)} & \rightarrow \frac{1}{n} \mathbb{E} \log \frac{V^{(n)}\left(Y^{n} \mid X^{n}\right)}{\tilde{P}^{(n)}\left(Y^{n}\right)} \\
& =\frac{1}{n} \mathbb{E} \log \frac{\mu^{(n)}\left(X^{n} \mid Y^{n}\right)}{P^{(n)}\left(X^{n}\right)} \\
& =\frac{1}{n}\left(I\left(X^{n} ; Y^{n}\right)-D\left(P^{(n)}\left(X^{n} \mid Y^{n}\right) \| \mu^{(n)}\left(X^{n} \mid Y^{n}\right)\right)\right) .
\end{aligned}
$$

where $\mu^{(n)}\left(X^{n} \mid Y^{n}\right)$ is the posterior probability induced by $\tilde{P}^{(n)} \times V^{(n)}$ and thus the divergence $D\left(P^{(n)}\left(X^{n} \mid Y^{n}\right) \| \mu^{(n)}\left(X^{n} \mid Y^{n}\right)\right)$ expresses an upper bound on the mismatch loss.

- Theorem 2 can also be extended to include lower bounded metrics in the following manner.

Corollary 2. Let $\boldsymbol{v}=\left\{v_{i}\right\}_{i \geq 1}$ be a lower bounded metrics sequence, i.e., $v_{n}\left(x^{n}, y^{n}\right) \geq-|B|>$ $-\infty, \forall n,\left(x^{n}, y^{n}\right) \in \mathcal{X}^{n} \times \mathcal{Y}^{n}$. The mismatch $\boldsymbol{v}$-capacity satisfies

$$
C \boldsymbol{v}(\boldsymbol{W}) \geq \sup _{\boldsymbol{P}} p-\liminf \frac{1}{n} \log \frac{v_{n}\left(X^{n}, Y^{n}\right)+|B|}{\sum_{x^{n} \in \mathcal{X}^{n}} P^{(n)}\left(x^{n}\right)\left[v_{n}\left(x^{n}, Y^{n}\right)+|B|\right]} .
$$

Proof: The proof follows by applying (36) to the non-negative metric $v_{n}\left(X^{n}, Y^{n}\right)+|B|$ which defines the same decision regions as those of $v_{n}\left(X^{n}, Y^{n}\right)$.

- The matched case: For a given sequence of input distributions $\boldsymbol{P}=\left\{P^{(i)}\right\}_{i \geq 1}$ and a channel $\boldsymbol{W}=\left\{W^{(i)}\right\}_{i \geq 1}$ recall the definitions of the inf-information rate and the sup-information rate [20] as

$$
\begin{aligned}
& \underline{I}(\boldsymbol{P}, \boldsymbol{W})=p-\liminf \frac{1}{n} \log \frac{W^{(n)}\left(Y^{n} \mid X^{n}\right)}{P_{Y^{n}}\left(Y^{n}\right)} \\
& \bar{I}(\boldsymbol{P}, \boldsymbol{W})=p-\limsup \frac{1}{n} \log \frac{W^{(n)}\left(Y^{n} \mid X^{n}\right)}{P_{Y^{n}}\left(Y^{n}\right)},
\end{aligned}
$$

respectively, where $\left(X^{n}, Y^{n}\right) \sim P^{(n)} \times W^{(n)}$ and consider the matched decoding metric

$$
q_{n}\left(x^{n}, y^{n}\right)=W^{(n)}\left(y^{n} \mid x^{n}\right)
$$

i.e., $q=W$. Note that

$$
\underline{I}(\boldsymbol{P}, \boldsymbol{W})=\underline{\Theta} \boldsymbol{W}(\boldsymbol{P}, \boldsymbol{W}),
$$

where $\Theta_{\boldsymbol{q}}(\boldsymbol{P}, \boldsymbol{W})$ is defined in (33) and $\Theta_{\boldsymbol{W}}(\boldsymbol{P}, \boldsymbol{W})=\left.\Theta_{\boldsymbol{q}}(\boldsymbol{P}, \boldsymbol{W})\right|_{\boldsymbol{q}=\boldsymbol{W}}$, i.e., $\boldsymbol{q}$ matches $\boldsymbol{W}$.

We emphasize the inequality relation between $\underline{\Theta} \boldsymbol{V}(\boldsymbol{P}, \boldsymbol{W})$ and $\underline{K}_{\boldsymbol{V}}(\boldsymbol{P}, \boldsymbol{W})$, when $\boldsymbol{V}$ is an MML metric, which is stated in the following lemma. Similarly to the definition of $\underline{K}_{\boldsymbol{q}}(\boldsymbol{W})$, define

$$
\bar{K}_{\boldsymbol{q}}(\boldsymbol{W})=\sup _{\boldsymbol{P}} \text { - } \limsup -\frac{1}{n} \log \left(\Phi_{q_{n}}\left(q_{n}\left(X^{n}, Y^{n}\right), P^{(n)}, Y^{n}\right)\right) \text {. }
$$


Lemma 2. For every channel $\boldsymbol{W}$, every MML decoding metrics sequence $\boldsymbol{V}$, and every sequence of distributions $\boldsymbol{P}$

$$
-\frac{1}{n} \log \left(\Phi_{V^{(n)}}\left(V^{(n)}\left(Y^{n} \mid X^{n}\right), P^{(n)}, Y^{n}\right)\right) \geq \frac{1}{n} \log \frac{V^{(n)}\left(Y^{n} \mid X^{n}\right)}{\tilde{P}^{(n)}\left(Y^{n}\right)},
$$

where $\tilde{P}^{(n)}$ is defined in (34) and consequently

$$
\underline{\Theta}_{\boldsymbol{V}}(\boldsymbol{P}, \boldsymbol{W}) \leq \underline{K}_{\boldsymbol{V}}(\boldsymbol{P}, \boldsymbol{W}),
$$

and

$$
\bar{\Theta}_{\boldsymbol{V}}(\boldsymbol{P}, \boldsymbol{W}) \leq \bar{K}_{\boldsymbol{V}}(\boldsymbol{P}, \boldsymbol{W}),
$$

Proof: The inequality (45) was derived in (37).

We note the following identity which stems from Theorem 1

Corollary 3. The following identity holds for every channel $\boldsymbol{W}=\left\{W^{(n)}\right\}_{n \geq 1}$,

$$
\sup _{\boldsymbol{P}} \underline{I}(\boldsymbol{P}, \boldsymbol{W})=\sup _{\boldsymbol{P}} \underline{K}_{\boldsymbol{W}}(\boldsymbol{P}, \boldsymbol{W}) .
$$

Proof: The left hand side of (48) is the general formula of the channel capacity in the matched case introduced by Verdú and Han [20]. From Theorem 1 it follows that the right hand side of (48) is equal to the capacity with matched decoding metric $\boldsymbol{W}$. Since (42) is nothing but the optimal ML decoding metric, it achieves capacity in the matched case and implies the equality (48). It should be noted that the optimal ML decoder breaks ties arbitrarily and the right hand side of (48) assumes that ties are considered as errors, but it is easily verified that considering ties as error does not reduce the achievable rate.

- We next state an important comment on the definition of the limit inferior in probability: Consider the equality (19) which states that for an $\left(n, M_{n}\right)$-code $\mathcal{C}_{n}$ we have

$$
\operatorname{Pr}\left\{-\frac{1}{n} \log \left(\Phi_{q_{n}}\left(q_{n}\left(X^{n}, Y^{n}\right), P^{(n)}, Y^{n}\right)\right)<\frac{1}{n} \log M_{n}\right\}=P_{e}\left(W^{(n)}, \mathcal{C}_{n}, q_{n}\right) .
$$

Note that it is easily verified that if one considers a loose inequality in 496, one has

$$
\operatorname{Pr}\left\{-\frac{1}{n} \log \left(\Phi_{q_{n}}\left(q_{n}\left(X^{n}, Y^{n}\right), P^{(n)}, Y^{n}\right)\right) \leq \frac{1}{n} \log M_{n}\right\}=1 .
$$

To realize this, recall 20], which yields that the right hand of 49) is equal to

$$
\begin{aligned}
& \operatorname{Pr}\left\{-\frac{1}{n} \log \left(\left|\left\{\boldsymbol{x}^{\prime} \in \mathcal{C}_{n}: q_{n}\left(\boldsymbol{x}^{\prime}, Y^{n}\right) \geq q_{n}\left(X^{n}, Y^{n}\right)\right\}\right|\right) \leq 0\right\} \\
= & \operatorname{Pr}\left\{\left|\left\{\boldsymbol{x}^{\prime} \in \mathcal{C}_{n}: q_{n}\left(\boldsymbol{x}^{\prime}, Y^{n}\right) \geq q_{n}\left(X^{n}, Y^{n}\right)\right\}\right| \geq 1\right\} \\
= & 1
\end{aligned}
$$

where the last step follows since by setting $\boldsymbol{x}^{\prime}=X^{n} \in \mathcal{C}_{n}$ we have $q_{n}\left(\boldsymbol{x}^{\prime}, Y^{n}\right)=q_{n}\left(X^{n}, Y^{n}\right)$.

Comparing (49) and (50) one realizes that the subtlety of a strict inequality in definition 8 (see (10)) is important in establishing the proof of Theorem 1 
Let a channel $W^{(n)}$ be given. The following theorem provides sufficient conditions for another channel, $\tilde{W}^{(n)}$, to have average probability of error essentially (up to a vanishing gap) no larger than that of $W^{(n)}$. It is a direct consequence of Lemma 1, and it will be useful in deriving upper bounds in the spirit of the general formula of the channel capacity in the matched case [20], and also in the derivation of the mismatch capacity of the DMC.

Theorem 3. Let $X^{n}$ be a random vector uniformly distributed over a codebook $\mathcal{C}_{n}$, let $W^{(n)}$ and $\tilde{W}^{(n)}$ be two channels from $\mathcal{X}^{n}$ to $\mathcal{Y}^{n}$, whose outputs when fed by $X^{n}$ are denoted $Y^{n}$ and $\tilde{Y}^{n}$, respectively. If there exist sequences $\zeta_{n} \geq 0, \eta_{n} \geq 0$, and $\tau_{n}, n \geq 1$ such that

$$
\begin{aligned}
& \operatorname{Pr}\left\{\tau_{n}<q_{n}\left(X^{n}, Y^{n}\right)\right\} \leq \zeta_{n} \\
& \operatorname{Pr}\left\{q_{n}\left(X^{n}, \tilde{Y}^{n}\right)<\tau_{n}\right\} \leq \eta_{n}
\end{aligned}
$$

and

$$
P_{\tilde{Y}^{n}}=P_{Y^{n}}
$$

then

$$
P_{e}\left(\tilde{W}^{(n)}, \mathcal{C}_{n}, q_{n}\right) \leq P_{e}\left(W^{(n)}, \mathcal{C}_{n}, q_{n}\right)+\zeta_{n}+\eta_{n}
$$

Proof: Let $P^{(n)}$ be uniform over $\mathcal{C}_{n}$ and denote

$$
E_{n}\left(q_{n}\left(X^{n}, Y^{n}\right), P^{(n)}, Y^{n}\right) \triangleq-\frac{1}{n} \log \left(\Phi_{q_{n}}\left(q_{n}\left(X^{n}, Y^{n}\right), P^{(n)}, Y^{n}\right)\right) .
$$

From Lemma 1 we know that

$$
\begin{aligned}
P_{e}\left(W^{(n)}, \mathcal{C}_{n}, q_{n}\right) & =\operatorname{Pr}\left\{E_{n}\left(q_{n}\left(X^{n}, Y^{n}\right), P^{(n)}, Y^{n}\right)<\frac{1}{n} \log M_{n}\right\} \\
& \geq \operatorname{Pr}\left\{E_{n}\left(q_{n}\left(X^{n}, Y^{n}\right), P^{(n)}, Y^{n}\right)<\frac{1}{n} \log M_{n}, \tau_{n} \geq q_{n}\left(X^{n}, Y^{n}\right)\right\} \\
& \stackrel{(a)}{\geq} \operatorname{Pr}\left\{E_{n}\left(\tau_{n}, P^{(n)}, Y^{n}\right)<\frac{1}{n} \log M_{n}, \tau_{n} \geq q_{n}\left(X^{n}, Y^{n}\right)\right\} \\
& \stackrel{(b)}{\geq} \operatorname{Pr}\left\{E_{n}\left(\tau_{n}, P^{(n)}, Y^{n}\right)<\frac{1}{n} \log M_{n}\right\}-\zeta_{n} \\
& \stackrel{(c)}{=} \operatorname{Pr}\left\{E_{n}\left(\tau_{n}, P^{(n)}, \tilde{Y}^{n}\right)<\frac{1}{n} \log M_{n}\right\}-\zeta_{n} \\
& \geq \operatorname{Pr}\left\{E_{n}\left(\tau_{n}, P^{(n)}, \tilde{Y}^{n}\right)<\frac{1}{n} \log M_{n}, q_{n}\left(X^{n}, \tilde{Y}^{n}\right) \geq \tau_{n}\right\}-\zeta_{n} \\
& \stackrel{(d)}{\geq} \operatorname{Pr}\left\{E_{n}\left(q_{n}\left(X^{n}, \tilde{Y}^{n}\right), P^{(n)}, \tilde{Y}^{n}\right)<\frac{1}{n} \log M_{n}, q_{n}\left(X^{n}, \tilde{Y}^{n}\right) \geq \tau_{n}\right\}-\zeta_{n} \\
& \stackrel{(e)}{\geq} \operatorname{Pr}\left\{E_{n}\left(q_{n}\left(X^{n}, \tilde{Y}^{n}\right), P^{(n)}, \tilde{Y}^{n}\right)<\frac{1}{n} \log M_{n}\right\}-\zeta_{n}-\eta_{n} \\
& =P_{e}\left(\tilde{W}^{(n)}, \mathcal{C}_{n}, q_{n}\right)-\zeta_{n}-\eta_{n},
\end{aligned}
$$

where $(a)$ and $(d)$ follow since $\Phi(c, \mu, \boldsymbol{y})$ is non increasing in $c,(b)$ follows from (52), $(c)$ follows from (54) and (e) follows from (53). 
Before we state an upper bound on the mismatch $\boldsymbol{q}$-capacity which stems from Theorem 3 we present the following definition.

Definition 11. For a given sequence of input distributions $\boldsymbol{P}=\left\{P^{(i)}\right\}_{i \geq 1}$ and a sequence of metrics $\boldsymbol{q}$, let $\mathcal{W}_{\boldsymbol{q}}(\boldsymbol{P}, \boldsymbol{W})$ be the set of channels $\tilde{\boldsymbol{W}}=\tilde{W}^{(n)}, n \geq 1$ such that

$$
\forall n, P_{Y^{n}}=P_{\tilde{Y}^{n}}
$$

and there exists a sequence $\tau_{n}, n \geq 1$ such that

$$
p-\liminf \left(q_{n}\left(X^{n}, \tilde{Y}^{n}\right)-\tau_{n}\right) \geq 0 \geq p-\lim \sup \left(q_{n}\left(X^{n}, Y^{n}\right)-\tau_{n}\right)
$$

where $\left(X^{n}, Y^{n}\right) \sim P^{(n)} \times W^{(n)}$ and $\left(X^{n}, \tilde{Y}^{n}\right) \sim P^{(n)} \times \tilde{W}^{(n)}$.

The following theorem presents an upper bound on the mismatch $\boldsymbol{q}$-capacity in terms of the supremum (over sequences of input distributions) of the infimum over channels of the mutual information density rates of $\left(X^{n}, \tilde{Y}^{n}\right)$ where $\tilde{Y}^{n}$ is the output process.

Theorem 4. The mismatch $\boldsymbol{q}$-capacity of the channel $\boldsymbol{W}$ is upper bounded as follows

$$
C_{\boldsymbol{q}}(\boldsymbol{W}) \leq \sup _{\boldsymbol{P} \in \mathcal{P}_{U}^{(\infty)}} \inf _{\boldsymbol{W} \in \mathcal{W}_{\boldsymbol{q}}(\boldsymbol{P}, \boldsymbol{W})} \underline{I}(\boldsymbol{P}, \tilde{\boldsymbol{W}})
$$

\section{Proof:}

Let $\left\{\mathcal{C}_{n}\right\}_{n \geq 1}$ be a given code sequence, let $M_{n}$ stand for the cardinality of $\mathcal{C}_{n}$, and let $\boldsymbol{P}=\left\{P^{(i)}\right\}_{i \geq 1}$ be the corresponding distributions of the input vectors, i.e., $P^{(n)}$ is uniform over $\mathcal{C}_{n}$. From [59], we have that if $\tilde{\boldsymbol{W}} \in \mathcal{W}_{\boldsymbol{q}}(\boldsymbol{P}, \boldsymbol{W})$ then there exist vanishing sequences $\zeta_{n}, \eta_{n} \geq 0$ such that the conditions (52)-(54) are met for some sequence $\tau_{n}$. Now, from Theorem 3, we obtain that for all $n$

$$
P_{e}\left(W^{(n)}, \mathcal{C}_{n}, q_{n}\right)+\zeta_{n}+\eta_{n} \geq P_{e}\left(\tilde{W}^{(n)}, \mathcal{C}_{n}, q_{n}\right)
$$

and consequently

$$
\limsup _{n \rightarrow \infty} P_{e}\left(W^{(n)}, \mathcal{C}_{n}, q_{n}\right) \geq \limsup _{n \rightarrow \infty} P_{e}\left(\tilde{W}^{(n)}, \mathcal{C}_{n}, q_{n}\right)
$$

From [20, Theorem 1], we have for the channel $\tilde{W}^{(n)}$

$$
P_{e}\left(\tilde{W}^{(n)}, \mathcal{C}_{n}, q_{n}\right) \geq \operatorname{Pr}\left\{\frac{1}{n} \log \frac{\tilde{W}^{(n)}\left(\tilde{Y}^{n} \mid X^{n}\right)}{P_{\tilde{Y}^{n}}\left(\tilde{Y}^{n}\right)} \leq \frac{1}{n} \log M_{n}-\gamma\right\}-e^{-n \gamma},
$$

where $\gamma>0$ is an arbitrary constant, and where in fact, this inequality holds also for the matched case with ML metric corresponding to $\tilde{W}^{(n)}$.

Hence, if

$$
R>p-\liminf \frac{1}{n} \log \frac{\tilde{W}^{(n)}\left(\tilde{Y}^{n} \mid X^{n}\right)}{P_{\tilde{Y}^{n}}\left(\tilde{Y}^{n}\right)},
$$

the average probability of error of the codebook sequence $\left\{\mathcal{C}_{n}\right\}_{n \geq 1}, P_{e}\left(\tilde{W}^{(n)}, \mathcal{C}_{n}, q_{n}\right)$, does not vanish, and from (62) neither does $P_{e}\left(W^{(n)}, \mathcal{C}_{n}, q_{n}\right)$. The proof of this claim follows similarly to equations (3.11)-(3.14) in [20]. 
Since the claim (64) holds for every $\tilde{\boldsymbol{W}} \in \mathcal{W}(\boldsymbol{P}, \boldsymbol{W})$, we have that if

$$
R>\inf _{\tilde{\boldsymbol{W}} \in \mathcal{W}_{\boldsymbol{q}}(\boldsymbol{P}, \boldsymbol{W})} p-\liminf \frac{1}{n} \log \frac{\tilde{W}^{(n)}\left(\tilde{Y}^{n} \mid X^{n}\right)}{P_{\tilde{Y}^{n}}\left(\tilde{Y}^{n}\right)},
$$

the average probability of error of the codebook sequence $\left\{\mathcal{C}_{n}\right\}_{n \geq 1}$ does not vanish.

The above derivation holds for a given sequence of codebooks $\left\{\mathcal{C}_{n}\right\}_{n \geq 1}$ and its corresponding $\boldsymbol{P}=\left\{P^{(n)}\right\}_{n \geq 1}$. Taking the supremum over $\boldsymbol{P} \in \mathcal{P}_{U}^{(\infty)}$ we obtain that if

$$
R>\sup _{\boldsymbol{P} \in \mathcal{P}_{U}^{(\infty)}} \inf _{\boldsymbol{W} \in \mathcal{W}_{\boldsymbol{q}}(\boldsymbol{P}, \boldsymbol{W})} p-\liminf \frac{1}{n} \log \frac{\tilde{W}^{(n)}\left(\tilde{Y}^{n} \mid X^{n}\right)}{P_{\tilde{Y}^{n}}\left(\tilde{Y}^{n}\right)},
$$

there exists no sequence of codebooks with vanishingly low average probability of error.

We next deduce an alternative upper bound on the general channel mismatch capacity which requires the following definition.

Definition 12. For a given sequence of input distributions $\boldsymbol{P}$, let $\mathcal{W}_{\boldsymbol{q}}^{\prime}(\boldsymbol{P}, \boldsymbol{W})$ be the set of channels $\tilde{\boldsymbol{W}}=\tilde{W}^{(n)}, n \geq$ 1 such that

$$
\forall n, P_{Y^{n}, q_{n}\left(X^{n}, Y^{n}\right)}=P_{\tilde{Y}^{n}, q_{n}\left(X^{n}, \tilde{Y}^{n}\right)},
$$

where $\left(X^{n}, Y^{n}\right) \sim P^{(n)} \times W^{(n)}$ and $\left(X^{n}, \tilde{Y}^{n}\right) \sim P^{(n)} \times \tilde{W}^{(n)}$.

In other words, $\mathcal{W}_{\boldsymbol{q}}^{\prime}(\boldsymbol{P}, \boldsymbol{W})$ is the set of channels which induce the same joint law of the channel output and the metric between the channel input and output as that induced by $W$.

Theorem 5. The mismatch $\boldsymbol{q}$-capacity of the channel $\boldsymbol{W}$ is upper bounded as follows

$$
C_{\boldsymbol{q}}(\boldsymbol{W}) \leq \sup _{\boldsymbol{P} \in \mathcal{P}_{U}^{(\infty)}} \inf _{\boldsymbol{W} \in \mathcal{W}_{\boldsymbol{q}}^{\prime}(\boldsymbol{P}, \boldsymbol{W})} \underline{I}(\boldsymbol{P}, \tilde{\boldsymbol{W}}) .
$$

Proof: The theorem follows by noting that for every codebook $\mathcal{C}_{n}$, one has $P_{e}\left(W^{(n)}, \mathcal{C}_{n}, q_{n}\right)=$ $P_{e}\left(\tilde{W}^{(n)}, \mathcal{C}_{n}, q_{n}\right)$ for every channel $\tilde{W}^{(n)}$ whose output $\tilde{Y}^{n}$ shares the same joint law with $q_{n}\left(X^{n}, \tilde{Y}^{n}\right)$ as that of $\left(Y^{n}, q_{n}\left(X^{n}, Y^{n}\right)\right)$, as the left hand side of $(19)$ is identical for both channels $W^{(n)}$ and $\tilde{W}^{(n)}$. Consequently, if $\tilde{\boldsymbol{W}} \in \mathcal{W}_{\boldsymbol{q}}^{\prime}(\boldsymbol{P}, \boldsymbol{W})$ one has

$$
\limsup _{n \rightarrow \infty} P_{e}\left(W^{(n)}, \mathcal{C}_{n}, q_{n}\right)=\limsup _{n \rightarrow \infty} P_{e}\left(\tilde{W}^{(n)}, \mathcal{C}_{n}, q_{n}\right) .
$$

The rest of the derivation follows along the line of the proof of Theorem 4 from the step proceeding (62) by substituting $\mathcal{W}_{\boldsymbol{q}}(\boldsymbol{P}, \boldsymbol{W})$ with $\mathcal{W}_{\boldsymbol{q}}^{\prime}(\boldsymbol{P}, \boldsymbol{W})$.

We note that it is not evident which of the upper bounds (of Theorem 4 or 5) is tighter since neither of the sets $\mathcal{W}_{\boldsymbol{q}}(\boldsymbol{P}, \boldsymbol{W}), \mathcal{W}_{\boldsymbol{q}}^{\prime}(\boldsymbol{P}, \boldsymbol{W})$ is a subset of the other. 


\section{The Threshold CAPACity}

In this section, we study the threshold capacity. We distinguish between two setups which will be referred to as: threshold decoding and constant threshold decoding. Recall the definition of a threshold decoder (5)-(6), and the proceeding Definitions 6, 7] of the threshold $\boldsymbol{q}$-capacity and the constant threshold $\boldsymbol{q}$-capacity of a channel $\boldsymbol{W}$. The following definition extends $P_{e}\left(W^{(n)}, \mathcal{C}_{n}, q_{n}\right)$ to the case of a threshold decoder,

Definition 13. For a given codebook $\mathcal{C}_{n}$, let $P_{e}\left(W^{(n)}, \mathcal{C}_{n},\left(q_{n}, \tau_{n}\right)\right)$ designate the average probability of error incurred by the $\left(q_{n}, \tau_{n}\right)$-threshold decoder employed on the output of the channel $W^{(n)}$.

We first prove the following straightforward lemma concerning threshold decoders.

Lemma 3. For every channel $W^{(n)},\left(n, M_{n}\right)$-codebook $\mathcal{C}_{n}$, mismatched decoder $q_{n}$, and threshold level $\tau_{n}$ one has

$$
P_{e}\left(W^{(n)}, \mathcal{C}_{n},\left(q_{n}, \tau_{n}\right)\right) \geq \frac{1}{2} P_{e}\left(W^{(n)}, \mathcal{C}_{n}, q_{n}\right)
$$

Proof: Let $X^{n}$ and $Y^{n}$ denote the input and output of the channel $W^{(n)}$, respectively, and let $\mathcal{C}_{n}=\left\{\boldsymbol{x}_{j}\right\}_{j=1}^{M_{n}}$. Recall that $S$ denotes the transmitted message, which is uniformly distributed over $\left\{1, \ldots, M_{n}\right\}$. The following inequalities hold:

$$
\begin{aligned}
& P_{e}\left(W^{(n)}, \mathcal{C}_{n},\left(q_{n}, \tau_{n}\right)\right) \\
&= \operatorname{Pr}\left(\left\{q_{n}\left(X^{n}, Y^{n}\right)<\tau_{n}\right\} \cup\left\{\exists j \neq S: q_{n}\left(\boldsymbol{x}_{j}, Y^{n}\right) \geq \tau_{n}, q_{n}\left(X^{n}, Y^{n}\right) \geq \tau_{n}\right\}\right) \\
& \stackrel{(a)}{\geq} \frac{1}{2}\left[\operatorname{Pr}\left(\left\{q_{n}\left(X^{n}, Y^{n}\right)<\tau_{n}\right\}\right)+\operatorname{Pr}\left(\exists j \neq S: q_{n}\left(\boldsymbol{x}_{j}, Y^{n}\right) \geq \tau_{n}, q_{n}\left(X^{n}, Y^{n}\right) \geq \tau_{n}\right)\right] \\
& \geq \frac{1}{2}\left[\operatorname{Pr}\left(\left\{q_{n}\left(X^{n}, Y^{n}\right)<\tau_{n}\right\}\right)+\operatorname{Pr}\left(\exists j \neq S: q_{n}\left(\boldsymbol{x}_{j}, Y^{n}\right) \geq q_{n}\left(X^{n}, Y^{n}\right), q_{n}\left(X^{n}, Y^{n}\right) \geq \tau_{n}\right)\right] \\
& \geq \frac{1}{2}\left[\operatorname{Pr}\left(\left\{q_{n}\left(X^{n}, Y^{n}\right)<\tau_{n}\right\}\right)+\operatorname{Pr}\left(\exists j \neq S: q_{n}\left(\boldsymbol{x}_{j}, Y^{n}\right) \geq q_{n}\left(X^{n}, Y^{n}\right)\right)+\operatorname{Pr}\left(q_{n}\left(X^{n}, Y^{n}\right) \geq \tau_{n}\right)-1\right] \\
&= \frac{1}{2} \cdot \operatorname{Pr}\left(\exists j \neq S: q_{n}\left(\boldsymbol{x}_{j}, Y^{n}\right) \geq q_{n}\left(X^{n}, Y^{n}\right)\right) \\
&= \frac{1}{2} P_{e}\left(W^{(n)}, \mathcal{C}_{n}, q_{n}\right),
\end{aligned}
$$

where $(a)$ follows since for two events $A, B$, one has $\operatorname{Pr}(A \cup B) \geq \max \{\operatorname{Pr}(A), \operatorname{Pr}(B)\} \geq \frac{1}{2}(\operatorname{Pr}(A)+\operatorname{Pr}(B))$.

Consequently, $P_{e}\left(W^{(n)}, \mathcal{C}_{n},\left(q_{n}, \tau_{n}\right)\right)$ cannot vanish as $n$ tends to infinity unless $P_{e}\left(W^{(n)}, \mathcal{C}_{n}, q_{n}\right)$ does, and the rate achieved by the threshold decoder $\left(q_{n}, \tau_{n}\right)$ cannot exceed the rate achieved by the decoder $q_{n}$.

The following lemma is the equivalent of Lemma 1 to the threshold decoding case, it will serve to prove the converse result.

Lemma 4. Let $X^{n}$ be the random variable uniformly distributed over an $\left(n, M_{n}\right)$-code $\mathcal{C}_{n}$, and $Y^{n}$ the output of 
a channel $W^{(n)}$ with $X^{n}$ as the input, then

$$
\begin{aligned}
& P_{e}\left(W^{(n)}, \mathcal{C}_{n},\left(q_{n}, \tau_{n}\right)\right) \\
= & \operatorname{Pr}\left\{\left\{-\frac{1}{n} \log \left(\Phi_{q_{n}}\left(\tau_{n}, P^{(n)}, Y^{n}\right)\right)<\frac{1}{n} \log M_{n}, q_{n}\left(X^{n}, Y^{n}\right) \geq \tau_{n}\right\} \cup\left\{q_{n}\left(X^{n}, Y^{n}\right)<\tau_{n}\right\}\right\},
\end{aligned}
$$

where $P^{(n)}$ is the distribution of the codeword $X^{n}$, i.e., a uniform distribution over $\mathcal{C}_{n}$.

The proof of the lemma follows similarly to that of Lemma 1 but it is included for the sake of completeness.

Proof: Note that

$$
\begin{aligned}
& \Phi_{q_{n}}\left(\tau_{n}, P^{(n)}, Y^{n}\right) \\
= & \sum_{\boldsymbol{x}^{\prime} \in \mathcal{C}_{n}: q_{n}\left(\boldsymbol{x}^{\prime}, Y^{n}\right) \geq \tau_{n}} P^{(n)}\left(\boldsymbol{x}^{\prime}\right) \\
= & \frac{\left|\left\{\boldsymbol{x}^{\prime} \in \mathcal{C}_{n}: q_{n}\left(\boldsymbol{x}^{\prime}, Y^{n}\right) \geq \tau_{n}\right\}\right|}{M_{n}}
\end{aligned}
$$

where the last equality follows since $X^{n}$ is distributed uniformly over the codebook of size $M_{n}$. Hence, the right hand side of (72) is equal to

$$
\begin{aligned}
& \operatorname{Pr}\left\{\left\{-\frac{1}{n} \log \left(\left|\left\{\boldsymbol{x}^{\prime} \in \mathcal{C}_{n}: q_{n}\left(\boldsymbol{x}^{\prime}, Y^{n}\right) \geq \tau_{n}\right\}\right|\right)<0, q_{n}\left(X^{n}, Y^{n}\right) \geq \tau_{n}\right\} \cup\left\{q_{n}\left(X^{n}, Y^{n}\right)<\tau_{n}\right\}\right\} \\
= & \operatorname{Pr}\left\{\left\{\left|\left\{\boldsymbol{x}^{\prime} \in \mathcal{C}_{n}: q_{n}\left(\boldsymbol{x}^{\prime}, Y^{n}\right) \geq \tau_{n}\right\}\right|>1, q_{n}\left(X^{n}, Y^{n}\right) \geq \tau_{n}\right\} \cup\left\{q_{n}\left(X^{n}, Y^{n}\right)<\tau_{n}\right\}\right\} \\
= & P_{e}\left(W^{(n)}, \mathcal{C}_{n},\left(q_{n}, \tau_{n}\right)\right),
\end{aligned}
$$

where the last step follows since the decision rule (5)-(6) can be rewritten as: decide $m$ iff

$$
\left|\left\{\boldsymbol{x}^{\prime} \in \mathcal{C}_{n}: q_{n}\left(\boldsymbol{x}^{\prime}, \boldsymbol{y}\right) \geq \tau_{n}\right\}\right|=1 \text { and } q_{n}\left(\boldsymbol{x}_{m}, \boldsymbol{y}\right) \geq \tau_{n}
$$

and the corresponding decision region $\mathcal{D}_{m}$ is

$$
\mathcal{D}_{m}=\left\{\boldsymbol{y}:\left|\left\{\boldsymbol{x}^{\prime} \in \mathcal{C}_{n}: q_{n}\left(\boldsymbol{x}^{\prime}, \boldsymbol{y}\right) \geq \tau_{n}\right\}\right|=1 \text { and }\left\{q_{n}\left(\boldsymbol{x}_{m}, \boldsymbol{y}\right) \geq \tau_{n}\right\}\right\}
$$

This concludes the proof of Lemma 4

Next, we obtain an asymptotic expression for $P_{e}\left(W^{(n)}, \mathcal{C}_{n},\left(q_{n}, \tau\right)\right)$ of a given sequence of codebooks and the corresponding $\boldsymbol{P}$, with constant threshold level $\tau<\tau_{\boldsymbol{q}}^{*}(\boldsymbol{P}, \boldsymbol{W})$ where

$$
\tau_{\boldsymbol{q}}^{*}(\boldsymbol{P}, \boldsymbol{W})=p-\liminf q_{n}\left(X^{n}, Y^{n}\right)
$$

Lemma 5. Let $\left\{\mathcal{C}_{n}\right\}_{n \geq 1}$ be a sequence of codes, where $\forall n, \mathcal{C}_{n}$ is an $\left(n, M_{n}\right)$-code and let $P^{(n)}$ be the uniform distribution over $\mathcal{C}_{n}$. Denote $\boldsymbol{P}=\left\{P^{(n)}\right\}_{n \geq 1}$, and let $\boldsymbol{W}=\left\{W^{(n)}\right\}_{n \geq 1}$ be a given channel. For all $\tau<\tau_{\boldsymbol{q}}^{*}(\boldsymbol{P}, \boldsymbol{W})$ one has

$$
\lim _{n \rightarrow \infty}\left|P_{e}\left(W^{(n)}, \mathcal{C}_{n},\left(q_{n}, \tau\right)\right)-\operatorname{Pr}\left\{-\frac{1}{n} \log \left(\Phi_{q_{n}}\left(\tau, P^{(n)}, Y^{n}\right)\right)<\frac{1}{n} \log M_{n}\right\}\right|=0
$$


where $\left(X^{n}, Y^{n}\right) \sim P^{(n)} \times W^{(n)}$.

Proof: By definition of the limit inferior in probability, since $\tau<\tau_{\boldsymbol{q}}^{*}(\boldsymbol{P}, \boldsymbol{W})$, there exists a vanishing sequence $\xi_{n}$ such that

$$
\operatorname{Pr}\left\{q_{n}\left(X^{n}, Y^{n}\right)<\tau\right\} \leq \xi_{n}, \forall n
$$

Consequently, from Lemma 4 on one hand,

$$
\begin{aligned}
P_{e}\left(W^{(n)}, \mathcal{C}_{n},\left(q_{n}, \tau\right)\right) & \leq \operatorname{Pr}\left\{-\frac{1}{n} \log \left(\Phi_{q_{n}}\left(\tau, P^{(n)}, Y^{n}\right)\right)<\frac{1}{n} \log M_{n}, q_{n}\left(X^{n}, Y^{n}\right) \geq \tau\right\}+\operatorname{Pr}\left\{q_{n}\left(X^{n}, Y^{n}\right)<\tau\right\} \\
& \leq \operatorname{Pr}\left\{-\frac{1}{n} \log \left(\Phi_{q_{n}}\left(\tau, P^{(n)}, Y^{n}\right)\right)<\frac{1}{n} \log M_{n}\right\}+\xi_{n}
\end{aligned}
$$

and on the other hand, from Lemma 4

$$
\begin{aligned}
P_{e}\left(W^{(n)}, \mathcal{C}_{n},\left(q_{n}, \tau\right)\right) & \geq \operatorname{Pr}\left\{-\frac{1}{n} \log \left(\Phi_{q_{n}}\left(\tau, P^{(n)}, Y^{n}\right)\right)<\frac{1}{n} \log M_{n}, q_{n}\left(X^{n}, Y^{n}\right) \geq \tau\right\} \\
& \geq \operatorname{Pr}\left\{-\frac{1}{n} \log \left(\Phi_{q_{n}}\left(\tau, P^{(n)}, Y^{n}\right)\right)<\frac{1}{n} \log M_{n}\right\}-\xi_{n} .
\end{aligned}
$$

From the above lemmas and discussion we deduce the expression for the general formula for the constant threshold capacity.

Theorem 6. The constant threshold capacity of the channel $\boldsymbol{W}$ with decoding metrics sequence $\boldsymbol{q}$ is given by

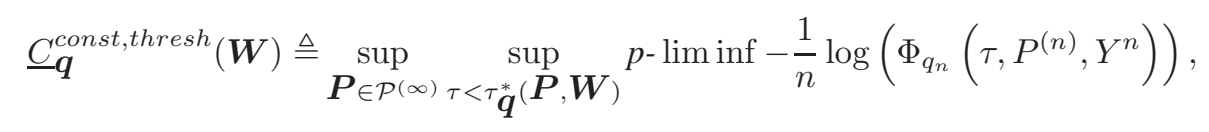

where $\boldsymbol{P}$ is a sequence of distributions $P^{(n)} \in \mathcal{P}\left(\mathcal{X}^{n}\right), n \geq 1,\left(X^{n}, Y^{n}\right) \sim P^{(n)} \times W^{(n)}$, and $\tau_{\boldsymbol{q}}^{*}(\boldsymbol{P}, \boldsymbol{W})$ is defined in (77). Further, the supremum can be restricted to $\boldsymbol{P} \in \mathcal{P}_{U}^{(\infty)}$.

Proof: From (72) it is clear that if one chooses a threshold level $\tau>\tau_{\boldsymbol{q}}^{*}(\boldsymbol{P}, \boldsymbol{W})$, one will have $P_{e}\left(W^{(n)}, \mathcal{C}_{n},\left(q_{n}, \tau\right)\right)$ bounded away from zero for infinitely many $n$ 's, i.e., $\lim _{\sup } P_{e}\left(W^{(n)}, \mathcal{C}_{n},\left(q_{n}, \tau\right)\right)>0$. We therefore compute the average probability of error using $\tau<\tau_{\boldsymbol{q}}^{*}(\boldsymbol{P}, \boldsymbol{W})$ arbitrarily close to $\tau_{\boldsymbol{q}}^{*}(\boldsymbol{P}, \boldsymbol{W})$. The proof of the converse part follows similarly to that of Theorem 10 with Lemma 5 replacing Lemma 1

It remains to prove the direct part of Theorem 6 The proof follows along the line of proof of the direct part of Theorem 1 with minor modifications, but we repeat it here for the sake of completeness.

Let $\boldsymbol{P}=\left\{P^{(n)}\right\}_{n \geq 1}$ be an arbitrary sequence of distributions where $P^{(n)} \in \mathcal{P}\left(\mathcal{X}^{n}\right)$. We employ random coding with $P^{(n)}$ to generate $M_{n}=2^{n R}$ independent codewords constituting the codebook where

$$
\begin{aligned}
R & =\left[\mathrm{p}-\liminf -\frac{1}{n} \log \left(\Phi_{q_{n}}\left(\tau, P^{(n)}, Y^{n}\right)\right)\right]-2 \gamma \\
& \triangleq D_{\boldsymbol{q}}(\boldsymbol{P}, \boldsymbol{W}, \tau)-2 \gamma,
\end{aligned}
$$

and $\gamma>0$ can be chosen arbitrarily small. Denote by $\tilde{\mathcal{A}}_{n}$ the set of pairs of $n$-vectors $(\boldsymbol{x}, \boldsymbol{y}) \in \mathcal{X}^{n} \times \mathcal{Y}^{n}$ such that $2^{n(D} \boldsymbol{q}^{(\boldsymbol{P}, \boldsymbol{W}, \tau)-\gamma)} \cdot \Phi_{q_{n}}\left(\tau, P^{(n)}, \boldsymbol{y}\right) \leq 1$. Note that by definition of $D_{\boldsymbol{q}}(\boldsymbol{P}, \boldsymbol{W}, \tau)$ we have $\operatorname{Pr}\left\{\tilde{\mathcal{A}}_{n}^{c}\right\} \rightarrow 0$ as 
$n$ tends to infinity. Note also that since $\tau<\tau_{\boldsymbol{q}}^{*}(\boldsymbol{P}, \boldsymbol{W})$, there exists a sequence $\zeta_{n}$ converging to zero such that $\operatorname{Pr}\left\{q_{n}\left(X^{n}, Y^{n}\right)<\tau\right\} \leq \zeta_{n}$. Further, the ensemble average probability of error can be computed as the probability of at least one "failure" in $M_{n}-1$ independent Bernoulli experiments, i.e., the average probability of error denoted $\bar{P}_{e}$ satisfies

$$
\begin{aligned}
& \bar{P}_{e} \leq \mathbb{E}\left[1-\left(1-\Phi_{q_{n}}\left(\tau, P^{(n)}, Y^{n}\right)\right)^{M_{n}-1}\right]+\operatorname{Pr}\left\{q_{n}\left(X^{n}, Y^{n}\right)<\tau\right\} \\
& \stackrel{(a)}{\leq} \mathbb{E} \min \left\{1, M_{n} \Phi\left(\tau, P^{(n)}, Y^{n}\right)\right\}+\zeta_{n} \\
& \leq \mathbb{E}\left(1\left\{\left(X^{n}, Y^{n}\right) \in \tilde{\mathcal{A}}_{n}\right\} \min \left\{1, M_{n} \Phi\left(\tau, P^{(n)}, Y^{n}\right)\right\}\right) \\
& +\operatorname{Pr}\left\{\tilde{\mathcal{A}}_{n}^{c}\right\}+\zeta_{n}
\end{aligned}
$$

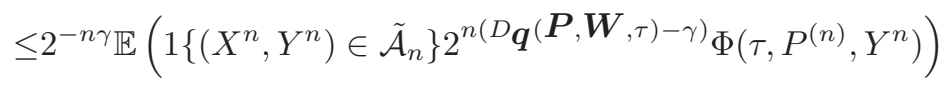

$$
\begin{aligned}
& +\operatorname{Pr}\left\{\tilde{\mathcal{A}}_{n}^{c}\right\}+\zeta_{n} \\
& \leq 2^{-n \gamma}+\operatorname{Pr}\left\{\tilde{\mathcal{A}}_{n}^{c}\right\}+\zeta_{n}
\end{aligned}
$$

where $\left(X^{n}, Y^{n}\right) \sim P^{(n)} \times W^{(n)}$, and $(a)$ follows from the union bound. Therefore, we have $\bar{P}_{e} \rightarrow 0$ as $n$ tends to infinity. The vanishing ensemble average probability of error ensures that there exists a sequence of deterministic codebooks of rate $R=D_{\boldsymbol{q}}(\boldsymbol{P}, \boldsymbol{W}, \tau)-2 \gamma$, whose average probability of error vanishes. The capacity formula follows since $\boldsymbol{P}$ is arbitrary, since $\gamma$ can be made arbitrarily small, and by definition of the capacity as the supremum of all achievable rates.

We next determine the expression for the threshold capacity.

Theorem 7. The threshold capacity of the channel $\boldsymbol{W}$ with decoding metrics sequence $\boldsymbol{q}$ is given by

$$
\underline{\boldsymbol{C}}_{\boldsymbol{q}}^{\text {thresh }}(\boldsymbol{W}) \triangleq \sup _{\boldsymbol{P} \in \mathcal{P}(\infty)} \sup _{\tau_{n}, n \geq 1} p \text { - } \liminf -\frac{1}{n} \log \left(\Phi_{q_{n}}\left(\tau_{n}, P^{(n)}, Y^{n}\right)\right)
$$

where $\boldsymbol{P}$ is a sequence of distributions $P^{(n)} \in \mathcal{P}\left(\mathcal{X}^{n}\right), n \geq 1,\left(X^{n}, Y^{n}\right) \sim P^{(n)} \times W^{(n)}$, and the supremum is over sequences $\tau_{n}, n \geq 1$ that satisfy

$$
\limsup _{n \rightarrow \infty} \operatorname{Pr}\left\{q_{n}\left(X^{n}, Y^{n}\right)<\tau_{n}\right\}=0 .
$$

Further, the supremum can be restricted to $\boldsymbol{P} \in \mathcal{P}_{U}^{(\infty)}$.

Proof: The direct part follows along the lines of the proof of Theorem 6 , substituting $\tau$ by $\tau_{n}$, and by (86). The converse part follows from (72) and since (86) implies that there exists a vanishing sequence $\zeta_{n}$ such that 
$\operatorname{Pr}\left\{q_{n}\left(X^{n}, Y^{n}\right)<\tau_{n}\right\} \leq \zeta_{n}$, and consequently

$$
\begin{aligned}
& \operatorname{Pr}\left\{\left\{-\frac{1}{n} \log \left(\Phi_{q_{n}}\left(\tau_{n}, P^{(n)}, Y^{n}\right)\right)<\frac{1}{n} \log M_{n}, q_{n}\left(X^{n}, Y^{n}\right) \geq \tau_{n}\right\} \cup q_{n}\left(X^{n}, Y^{n}\right)<\tau_{n}\right\} \\
\geq & \operatorname{Pr}\left\{-\frac{1}{n} \log \left(\Phi_{q_{n}}\left(\tau_{n}, P^{(n)}, Y^{n}\right)\right)<\frac{1}{n} \log M_{n}, q_{n}\left(X^{n}, Y^{n}\right) \geq \tau_{n}\right\} \\
\geq & \operatorname{Pr}\left\{-\frac{1}{n} \log \left(\Phi_{q_{n}}\left(\tau_{n}, P^{(n)}, Y^{n}\right)\right)<\frac{1}{n} \log M_{n}\right\}-\zeta_{n} .
\end{aligned}
$$

Additionally, the supremum over $\tau_{n}, n \geq 1$ in 85 is constrained by (86) since (72) implies that 86 is a necessary condition for vanishing average probability of error. To realize this, note that the left hand side of (72) satisfies:

$$
\begin{aligned}
& \operatorname{Pr}\left\{\left\{-\frac{1}{n} \log \left(\Phi_{q_{n}}\left(\tau_{n}, P^{(n)}, Y^{n}\right)\right)<\frac{1}{n} \log M_{n}, q_{n}\left(X^{n}, Y^{n}\right) \geq \tau_{n}\right\} \cup\left\{q_{n}\left(X^{n}, Y^{n}\right)<\tau_{n}\right\}\right\} \\
\geq & \operatorname{Pr}\left\{q_{n}\left(X^{n}, Y^{n}\right)<\tau_{n}\right\} .
\end{aligned}
$$

We observe that for every channel there exists a (matched) threshold decoder which is capacity achieving.

Proposition 1. For every channel $\boldsymbol{W}$ there exists a threshold decoder which achieves the matched capacity.

Proof: Consider the decoding rule (see [20]): decide $i$ iff $\boldsymbol{x}_{i}$ is the unique vector satisfying

$$
\frac{1}{n} \log \frac{W^{(n)}\left(\boldsymbol{y} \mid \boldsymbol{x}_{i}\right)}{P_{Y^{n}}(\boldsymbol{y})} \geq \underline{I}(\boldsymbol{P}, \boldsymbol{W})-\gamma .
$$

This is a threshold decoder w.r.t. the metric $q_{n}\left(x^{n}, y^{n}\right)=\frac{1}{n} \log \frac{W^{(n)}\left(y^{n} \mid x^{n}\right)}{P_{Y^{n}}\left(y^{n}\right)}$. It was used by Verdú and Han [20] to prove the direct part of the general channel capacity formula is capacity achieving for every channel.

We conclude this section with an example. Consider the erasures-only capacity of the channel $\boldsymbol{W}$, denoted $C_{e o}(\boldsymbol{W})$, which can be considered as the supremum of rates achievable by decoding with respect to the metric

$$
q_{n}^{e o}\left(x^{n}, y^{n}\right)=1\left\{W^{(n)}\left(y^{n} \mid x^{n}\right)>0\right\}
$$

with finite input and output alphabets. In this special case, it always holds that the actual transmitted codeword $X^{n}$ and received signal $Y^{n}$ satisfy

$$
q_{n}^{e o}\left(X^{n}, Y^{n}\right)=1
$$

Therefore, the erasures-only capacity $C_{e o}(\boldsymbol{W})$ is equal to the threshold capacity with fixed threshold level $\tau=1$. We obtain from Lemma 4

$$
\left.P_{e}\left(W^{(n)}, \mathcal{C}_{n},\left(q_{n}, \tau_{n}\right)\right)\right|_{\tau_{n}=1}=\operatorname{Pr}\left\{-\frac{1}{n} \log \left(\Phi_{q_{n}^{e o}}\left(1, P^{(n)}, Y^{n}\right)\right)<\frac{1}{n} \log M_{n}\right\},
$$

and consequently, the erasures-only capacity is stated in the following proposition. 
Proposition 2. The erasures-only capacity of the finite input and output alphabet channel $\boldsymbol{W}$ is given by

$$
\begin{aligned}
C_{e o}(\boldsymbol{W}) & =\sup _{\boldsymbol{P} \in \mathcal{P}(\infty)} p-\liminf -\frac{1}{n} \log \left(\Phi_{q_{n}^{e o}}\left(1, P^{(n)}, Y^{n}\right)\right) \\
& =\sup _{\boldsymbol{P} \in \mathcal{P}(\infty)} p-\liminf -\frac{1}{n} \log \left(\sum_{\tilde{x}^{n}: W^{(n)}\left(Y^{n} \mid \tilde{x}^{n}\right)>0} P^{(n)}\left(\tilde{x}^{n}\right)\right),
\end{aligned}
$$

where the supremum can be restricted to $\left\{\boldsymbol{P} \in \mathcal{P}_{U}^{(\infty)}\right\}$.

\section{The Mismatch Capacity of the DMC and Related Special Cases}

We next address the important special case of a DMC with mismatched decoding. We focus on bounded additive metrics $q_{n}$. Consider a DMC with a finite input alphabet $\mathcal{X}$ and finite output alphabet $\mathcal{Y}$, which is governed by the conditional p.m.f. $W$. As the channel is fed by an input vector $\boldsymbol{x} \in \mathcal{X}^{n}$, it generates an output vector $\boldsymbol{y} \in \mathcal{Y}^{n}$ according to the sequence of conditional probability distributions

$$
P\left(y_{i} \mid x_{1}, \ldots, x_{i}, y_{1}, \ldots, y_{i-1}\right)=W\left(y_{i} \mid x_{i}\right), \quad i=1,2, \ldots, n
$$

where for $i=1,\left(y_{1}, \ldots, y_{i-1}\right)$ is understood as the null string.

A special class of decoders is the class of additive decoding functions, i.e.,

$$
q_{n}\left(x^{n}, y^{n}\right)=\frac{1}{n} \sum_{i=1}^{n} q\left(x_{i}, y_{i}\right)
$$

where $q$ is a mapping from $\mathcal{X} \times \mathcal{Y}$ to $\mathbb{R}$. It will be assumed that $q$ is bounded, that is

$$
|q(x, y)| \leq B<\infty, \forall x \in \mathcal{X}, y \in \mathcal{Y} .
$$

As mentioned in the introduction, it was proved in [2] and [3] that the mismatch $q^{n}$-capacity of the DMC, denoted $C_{q}(W)$, is lower bounded by

$$
C_{q}^{(1)}(W)=\max _{P_{X}} \min _{\tilde{P} \in \mathcal{W}_{1}\left(P_{X}, W\right)} I_{\tilde{P}}(X ; \tilde{Y}),
$$

where for $P_{X} \in \mathcal{P}(\mathcal{X}), \mathcal{W}_{1}\left(P_{X}, W\right)$ is the following class of conditional probability distributions $\tilde{W}$ from $\mathcal{X}$ to $\mathcal{Y}$

$$
\mathcal{W}_{1}\left(P_{X}, W\right)=\left\{\tilde{W}: \tilde{P}_{Y}=P_{Y}, E_{\tilde{P}}(q(X, \tilde{Y})) \geq E_{P}(q(X, Y))\right\}
$$

$P=P_{X} \times W, \tilde{P}=P_{X} \times \tilde{W}$ and $(X, \tilde{Y}) \sim P_{X} \times \tilde{W}$. As mentioned in [1], by considering the achievable rate for the channel $W^{n}$ from $\mathcal{X}^{n}$ to $\mathcal{Y}^{n}$, the following rate is also achievable

$$
C_{q}^{(n)}(W)=\max _{P^{(n)}} \min _{\tilde{W}^{(n)} \in \mathcal{W}_{n}\left(P^{(n)}, W^{n}\right)} \frac{1}{n} I_{\tilde{P}}\left(X^{n} ; \tilde{Y}^{n}\right),
$$

where for $P^{(n)} \in \mathcal{P}\left(\mathcal{X}^{n}\right), \mathcal{W}_{n}\left(P^{(n)}, W^{n}\right)$ is the following class of conditional probability distributions $\tilde{W}^{(n)}$ from $\mathcal{X}^{n}$ to $\mathcal{Y}^{n}$

$$
\begin{gathered}
\mathcal{W}_{n}\left(P^{(n)}, W^{n}\right)=\left\{\tilde{W}^{(n)}: \tilde{P}_{Y^{n}}=P_{Y^{n}}, E_{\tilde{P}}\left(q_{n}\left(X^{n}, \tilde{Y}^{n}\right)\right) \geq E_{P}\left(q_{n}\left(X^{n}, Y^{n}\right)\right)\right\}, \\
P=P^{(n)} \times W^{n}, \tilde{P}=P^{(n)} \times \tilde{W}^{(n)} \text {, and }\left(X^{n}, \tilde{Y}^{n}\right) \sim P^{(n)} \times \tilde{W}^{(n)} .
\end{gathered}
$$


Theorem 8. The mismatch capacity of the DMC W with bounded additive decoding metric $q$ (95)-(96) is given by

$$
C_{q}^{(\infty)}(W) \triangleq \sup _{n \geq 1} C_{q}^{(n)}(W) .
$$

The proof of Theorem 8 appears in Appendix $\triangle$ it is divided into 9 steps which are outlined as follows:

1) Without loss of asymptotic optimality in terms of achievable rates, one can assume that the codebook contains codewords that lie in a single type-class.

2) Applying an $\left(n, M_{n}, \epsilon_{n}\right)$-code $\mathcal{C}_{n}$ repeatedly $K_{n}=o\left(\epsilon_{n}^{-1}\right)$ times over the DMC results in average probability of error not exceeding $K_{n} \times \epsilon_{n} \triangleq \bar{\epsilon}_{N}$, and thus one can analyze the performance of the concatenated codebook $\mathcal{C}_{N}^{\text {prod }}$, whose rate is equal to that of $\mathcal{C}_{n}$.

3) All the codewords of $\mathcal{C}_{N}^{\text {prod }}$ lie in a single type-class of sequences of length $N=n K_{n}$.

4) Let $X^{N}$ be the output of the encoder which uses the concatenated codebook $\mathcal{C}_{N}^{\text {prod }}$ and let $Y^{N}$ be the output of the DMC channel $W^{N}$ when fed by $X^{N}$, then if $q$ is bounded, for all $\Delta>0$

$$
\operatorname{Pr}\left\{q_{N}\left(X^{N}, Y^{N}\right) \geq \mathbb{E}_{P^{(N)} \times W^{(N)}}\left(q_{N}\right)+\Delta\right\} \leq \epsilon_{2, N},
$$

where $\epsilon_{2, N} \rightarrow 0$ as $N$ tends to infinity.

5) Let $\tilde{W}^{(N)}$ be a channel which satisfies

$$
\begin{aligned}
& \operatorname{Pr}\left\{q_{N}\left(X^{N}, \tilde{Y}^{(N)}\right) \leq \mathbb{E}\left(q_{N}\left(X^{N}, Y^{N}\right)\right)+\Delta\right\} \leq \epsilon_{1, N} \\
& P_{Y^{N}}=P_{\tilde{Y}^{N}},
\end{aligned}
$$

for some $\Delta>0, \epsilon_{1, N}>0$, where $\tilde{Y}^{N}$ is the output of $\tilde{W}^{(N)}$ when fed by $X^{N}$. Note that (102)-(104) are in fact the conditions (52)-(54) required for Theorem 3 to hold, and hence,

$$
P_{e}\left(\tilde{W}^{(N)}, \mathcal{C}_{N}^{\text {prod }}, q_{N}\right) \leq P_{e}\left(W^{N}, \mathcal{C}_{N}^{\text {prod }}, q_{N}\right)+\epsilon_{1, N}+\epsilon_{2, N} .
$$

6) From (105), it follow that we can invoke Fano's Inequality for the channel $\tilde{W}^{(N)}$ and this yields for all $\Delta>0$, $\epsilon>0$, and $N$ sufficiently large,

$$
R \leq \min _{\tilde{W}^{(N)} \in \mathcal{W}_{b}\left(P^{(N)}, \epsilon_{N, 1}\right)} \frac{1}{N} I\left(X^{N} ; \tilde{Y}^{N}\right)+R\left(\bar{\epsilon}_{N}+\epsilon_{1, N}+\epsilon_{2, N}\right)+\frac{1}{N},
$$

where $\left(X^{N}, \tilde{Y}^{N}\right) \sim P^{(N)} \times \tilde{W}^{(N)}$, and $\mathcal{W}_{b}\left(P^{(n)}, \epsilon_{N, 1}\right)$ is the set of channels which satisfy 103)-104).

7) Let $X^{N}=\left(\boldsymbol{X}^{(1)}, \ldots, \boldsymbol{X}^{\left(K_{n}\right)}\right)$ be a blockwise-memoryless source, i.e., $X^{n} \sim P^{(N)}=\prod_{k=0}^{K_{n}-1} \boldsymbol{P}^{(k)}$. For $\delta>\Delta$, one can show using the law of large numbers that $\mathcal{W}_{b}\left(P^{(N)}, \epsilon_{1, N}\right) \supseteq \mathcal{W}_{a}\left(P^{(N)}, \delta\right)$ where $\epsilon_{1, N}=\frac{B^{2}}{K_{n}(\delta-\Delta)}, B$ is the bound on $q$ (96), and $\mathcal{W}_{a}\left(P^{(N)}, \delta\right)$ is the set of block-wise memoryless channels $\tilde{W}^{(N)}=\prod_{k=0}^{K_{n}-1} \tilde{\boldsymbol{W}}^{(k)}$ (where $\tilde{\boldsymbol{W}}^{(k)}$ is a channel from $\mathcal{Y}^{n}$ to $\mathcal{X}^{n}$ whose input and output are $\boldsymbol{X}^{(k)}, \tilde{\boldsymbol{Y}}^{(k)}$, respectively) satisfying the constraints: $\forall k, \mathbb{E}_{\boldsymbol{P}^{(k)} \times \tilde{\boldsymbol{W}}^{(k)}} q_{n}\left(\boldsymbol{X}^{(k)}, \tilde{\boldsymbol{Y}}^{(k)}\right) \geq c^{(k)}+\delta, P_{\tilde{\boldsymbol{Y}}^{(k)}}=P_{\boldsymbol{Y}^{(k)}}$ where

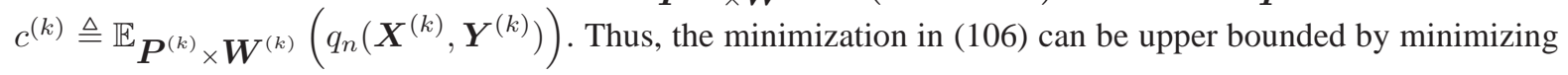
over $\mathcal{W}_{a}\left(P^{(N)}, \delta\right)$. 
8) Since $\tilde{W}^{(N)} \in \mathcal{W}_{a}\left(\prod_{k=0}^{K_{n}-1} \boldsymbol{P}^{(k)}, \delta\right)$ is block-wise memoryless, $\frac{1}{N} I\left(X^{N} ; \tilde{Y}^{N}\right) \leq \sum_{k=0}^{K_{n}-1} \frac{1}{N} I\left(\boldsymbol{X}^{(k)} ; \tilde{\boldsymbol{Y}}^{(k)}\right)$. It remains to maximize over the distribution of $X^{N}$ induced by the concatenated codebook $\mathcal{C}_{N}^{\text {prod }}$, and hence, the optimizations are in fact performed over block-wise memoryless sources and channels, and this yields

$$
R-\epsilon \leq \sup _{n} \max _{P^{(n)}} \min _{\tilde{W}^{(n)} \in \mathcal{W}_{n}\left(P^{(n)}, W^{(n)}, \delta\right)} \frac{1}{n} I\left(X^{n} ; \tilde{Y}^{n}\right) \triangleq C_{\boldsymbol{q}}(\boldsymbol{W}, \delta)
$$

for all $\epsilon>0$ and $n$ sufficiently large, where

$$
\mathcal{W}_{n}\left(P^{(n)}, W^{(n)}, \delta\right) \triangleq\left\{\tilde{W}^{(n)}: \mathbb{E}_{P^{(n)} \times \tilde{W}^{(n)}}\left(q_{n}\right) \geq \mathbb{E}_{P^{(n)} \times W^{(n)}}\left(q_{n}\right)+\delta, P_{\tilde{Y}^{n}}=P_{Y^{n}}\right\} .
$$

9) The case in which the set $\mathcal{W}_{n}\left(P^{(n)}, W^{(n)}, \delta\right)$ is empty is treated in Step 9. Finally, we take the limit as $\delta \rightarrow 0$, which yields the desired result.

The difference between $C_{q}^{(\infty)}(W)$ and the upper bounds given in Theorems 4 and 5 is twofold: a) the set over which the minimization is performed and b) the mutual information between $X^{n}$ and $\tilde{Y}^{n}$ rather than mutual information density rate of $\left(X^{n}, \tilde{Y}^{n}\right)$. In fact, it can be shown that the bound given in Theorem 4 is equal to $C_{q}^{(\infty)}(W)$ in the case of the DMC $W$, similarly to the proof of Theorem 8 ,

We note that the proof of the upper bound of Theorem 8 continues to hold for a larger class of channels rather than DMCs with bounded additive metrics. By inspecting the proof of the upper bound of Theorem 8 one realizes that the only steps in which the fact that the channel is memoryless and the metric is additive and bounded is used are Steps 2, 4, and 8. Roughly speaking, sufficient conditions for establishing a similar upper bound are:

- The channel $\boldsymbol{W}=W^{(n)}, n \geq 1$ is stationary and has a decaying memory in the sense that if $\mathcal{C}_{n}$ is an $\left(n, 2^{n R}, \epsilon_{n}\right)$-code, then applying $\mathcal{C}_{n}$ repeatedly $K_{n}=o\left(\epsilon_{n}^{-1}\right)$ times over the channel $W^{\left(n K_{n}\right)}$ results in average probability of error not exceeding $K_{n} \times \epsilon_{n}$.

- The condition that for every sequence of distributions $\boldsymbol{P}$ such that $P^{(n)} \in \mathcal{P}_{C C}(\mathcal{X}, n), \forall n$ (see the definition in (11) one has 4

$$
p-\liminf \left(q_{n}\left(X^{n}, Y^{n}\right)-\mathbb{E}_{P^{(n)} \times W^{(n)}}\left(q_{n}\right)\right) \leq 0 .
$$

- The condition that $C_{\boldsymbol{q}}(\boldsymbol{W}, \delta)$ (see (107)) is continuous in $\delta$ at the point $\delta=0$.

Under these conditions, the mismatch capacity is upper bounded by $\lim _{\delta \rightarrow 0} C_{\boldsymbol{q}}(\boldsymbol{W}, \delta)$.

Consider the case in which $\boldsymbol{q}$ is such that for all $n, x^{n}, y^{n}$, the function $q_{n}\left(x^{n}, y^{n}\right)$ depends only on the joint type-class of $x^{n}$ and $y^{n}([2])$. In this case, we denote with a little abuse of notation $q_{n}\left(x^{n}, y^{n}\right)=q_{n}\left(\hat{P}_{x^{n}}, y^{n}\right)$, where $\hat{P}_{x^{n}, y^{n}}$ is the joint empirical distribution induced by $\left(x^{n}, y^{n}\right)$. We shall refer to $\boldsymbol{q}$ that satisfies this condition as a type-dependent $\boldsymbol{q}$. An important thing to notice is that when $\boldsymbol{q}$ is type-dependent, the mismatch capacity takes

\footnotetext{
${ }^{4}$ This is a sufficient for Step 4 to hold.
} 
a special form. Note that

$$
\begin{aligned}
\Phi_{q_{n}}(c, \mu, \boldsymbol{y}) & =\sum_{\tilde{\boldsymbol{x}} \in \mathcal{X}^{n}: q_{n}(\tilde{\boldsymbol{x}}, \boldsymbol{y}) \geq c} \mu(\tilde{\boldsymbol{x}}) \\
& =\sum_{\hat{P}_{\tilde{\boldsymbol{x}} \mid \boldsymbol{y}}: q_{n}\left(\hat{P}_{\tilde{\boldsymbol{x}}, \boldsymbol{y}}\right) \geq c} \mu\left(T\left(\hat{P}_{\tilde{\boldsymbol{x}} \mid \boldsymbol{y}}\right)\right) \\
& \doteq \max _{\hat{P}_{\tilde{\boldsymbol{x}} \mid \boldsymbol{y}}: q_{n}\left(\hat{P}_{\tilde{\boldsymbol{x}}, \boldsymbol{y}}\right) \geq c} \mu\left(T\left(\hat{P}_{\tilde{\boldsymbol{x}} \mid \boldsymbol{y}}\right)\right),
\end{aligned}
$$

where the last equality on the exponential scale follows since the number of types is polynomial in $n$. Moreover, from Step 1 of the proof of Theorem 8, it follows that without loss of asymptotic optimality, one can assume that all the codewords of the codebook lie in a single type-class. This yields the following corollary.

Corollary 4. The mismatch capacity of the finite alphabet channel $\boldsymbol{W}$ with type-dependent decoding metrics sequence $\boldsymbol{q}$ is given by

$$
\begin{aligned}
& C_{\boldsymbol{q}}(\boldsymbol{W})=\sup _{\boldsymbol{P} \in \mathcal{P}(\infty)} p-\liminf -\frac{1}{n} \log \left(\max _{\tilde{P} \in \mathcal{P}_{n}(\mathcal{X} \times \mathcal{Y}): \tilde{P}_{Y}=\hat{P}_{Y^{n}}, q_{n}(\tilde{P}) \geq q_{n}\left(\hat{P}_{\left.X^{n}, Y^{n}\right)}\right.} P^{(n)}\left(T\left(\tilde{P}_{X, Y} \mid Y^{n}\right)\right)\right) \\
= & \sup _{\boldsymbol{P}: \forall n, P^{(n)} \in \mathcal{P}_{C C}(\mathcal{X}, n)} p-\liminf -\frac{1}{n} \log \left(\max _{\tilde{P} \in \mathcal{P}_{n}(\mathcal{X} \times \mathcal{Y}): \tilde{P}_{Y}=\hat{P}_{Y^{n}}, \tilde{P}_{X}=\hat{P}_{X^{n}, q_{n}(\tilde{P}) \geq q_{n}\left(\hat{P}_{\left.X^{n}, Y^{n}\right)}\right.}} P^{(n)}\left(T\left(\tilde{P}_{X, Y} \mid Y^{n}\right)\right)\right),
\end{aligned}
$$

where $\left(X^{n}, Y^{n}\right) \sim P^{(n)} \times W^{(n)}, T\left(\tilde{P}_{X, Y} \mid Y^{n}\right)$ is the set of $x^{n} \in \mathcal{X}^{n}$ whose joint empirical statistics with $Y^{n}$ is given by $\tilde{P}_{X, Y}$, and $\hat{P}_{X^{n}, Y^{n}}$ is the empirical distribution induced by $\left(X^{n}, Y^{n}\right)$.

Next, we deduce the following identity in the DMC case where $W^{(n)}=W^{n}$ and $q_{n}$ is given in (95).

Lemma 6. The following identity holds for a DMC W with a bounded additive decoding metric $q_{n}$ (95)

$$
C_{q}^{(\infty)}(W)=\sup _{\boldsymbol{P} \in \mathcal{P}(\infty)} \sup _{\epsilon>0} p \text { - } \lim \inf -\frac{1}{n} \log \left(\Phi_{q_{n}}\left(\mathbb{E}\left(q_{n}\left(X^{n}, Y^{n}\right)\right)-\epsilon, P^{(n)}, Y^{n}\right)\right),
$$

where $C_{q}^{(\infty)}(W)$ is defined in (101), $\left(X^{n}, Y^{n}\right) \sim P^{(n)} \times W^{(n)}$ and the supremum can be restricted to $\left\{\boldsymbol{P} \in \mathcal{P}_{U}^{(\infty)}\right\}$.

Proof: From Theorem 7 it follows that the r.h.s. of (112) is the rate achievable by threshold decoding with threshold level $\tau_{n}^{*}=\mathbb{E}_{P^{(n)} \times W^{n}}\left(q_{n}\left(X^{n}, Y^{n}\right)\right)-\epsilon$ for $\epsilon>0$ arbitrarily small. Lemma 3 and Theorem 8 imply that the r.h.s. of (112) is lower than $C_{q}^{(\infty)}(W)$.

To prove the opposite inequality, we pick an empirical distribution $P \in \mathcal{P}_{n}\left(\mathcal{X}^{n}\right)$ and use random coding uniform in $T(P)$, i.e., $P^{(n)}\left(x^{n}\right)=\frac{1\left\{x^{n} \in T(P)\right\}}{|T(P)|}$. Assume without loss of generality that the transmitted message $S$ is $i$ and denote the random codebook $\mathcal{C}=\left\{X^{n}(m)\right\}_{m=1}^{M_{n}}$. Fix an arbitrarily small $\epsilon>0$, and set $\tau_{n}^{*}=\mathbb{E}_{P^{(n)} \times W^{(n)}}\left(q_{n}\left(X^{n}, Y^{n}\right)\right)-\epsilon$. Let $X^{n}$ and $Y^{n}$ denote the input and output of the channel $W^{(n)}$, respectively. On one hand, the ensemble average 
probability of error, denoted $P_{e}\left(W^{(n)},\left(q_{n}, \tau_{n}^{*}\right)\right)$, satisfies

$$
\begin{aligned}
& P_{e}\left(W^{(n)},\left(q_{n}, \tau_{n}^{*}\right)\right) \\
&= \mathbb{E}\left(1-\left(1-\Phi_{q_{n}}\left(\tau_{n}^{*}, P^{(n)}, Y^{n}\right)\right)^{M_{n}}\right) \\
& \stackrel{(a)}{\geq} \frac{1}{2} \mathbb{E} \min \left\{1, M_{n} \Phi_{q_{n}}\left(\tau_{n}^{*}, P^{(n)}, Y^{n}\right)\right\} \\
& \geq \frac{1}{2} \operatorname{Pr}\left\{M_{n} \Phi_{q_{n}}\left(\tau_{n}^{*}, P^{(n)}, Y^{n}\right)>1\right\},
\end{aligned}
$$

where $(a)$ follows from [23, Lemma 1] stating that for $a \in[0,1]$, one has

$$
\frac{1}{2} \min \{1, M a\} \leq 1-[1-a]^{M} \leq \min \{1, M a\} .
$$

On the other hand assuming without loss of generality that the transmitted message is $i$, we have

$$
\begin{aligned}
& P_{e}\left(W^{(n)},\left(q_{n}, \tau_{n}^{*}\right)\right) \\
= & \operatorname{Pr}\left(\left\{q_{n}\left(X^{n}, Y^{n}\right)<\tau_{n}^{*}\right\} \cup\left\{\exists j \neq i: q_{n}\left(X^{n}(j), Y^{n}\right) \geq \tau_{n}^{*}, q_{n}\left(X^{n}, Y^{n}\right) \geq \tau_{n}^{*}\right\}\right) \\
\leq & \operatorname{Pr}\left(\left\{q_{n}\left(X^{n}, Y^{n}\right)<\tau_{n}^{*}\right\}\right)+\operatorname{Pr}\left(\exists j \neq i: q_{n}\left(X^{n}(j), Y^{n}\right) \geq \tau_{n}^{*}, q_{n}\left(X^{n}, Y^{n}\right) \geq \tau_{n}^{*}\right) \\
= & \operatorname{Pr}\left(\left\{q_{n}\left(X^{n}, Y^{n}\right)<\tau_{n}^{*}\right\}\right)+\operatorname{Pr}\left(\exists j \neq i: q_{n}\left(X^{n}(j), Y^{n}\right) \geq \tau_{n}^{*}, q_{n}\left(X^{n}, Y^{n}\right) \in\left[\tau_{n}^{*}, \tau_{n}^{*}+2 \epsilon\right]\right) \\
& +\operatorname{Pr}\left(\exists j \neq i: q_{n}\left(X^{n}(j), Y^{n}\right) \geq \tau_{n}^{*}, q_{n}\left(X^{n}, Y^{n}\right) \geq \tau_{n}^{*}+2 \epsilon\right) \\
\leq & \operatorname{Pr}\left(\left\{q_{n}\left(X^{n}, Y^{n}\right)<\tau_{n}^{*}\right\}\right)+\operatorname{Pr}\left(\exists j \neq i: q_{n}\left(X^{n}(j), Y^{n}\right) \geq q_{n}\left(X^{n}, Y^{n}\right)-2 \epsilon\right) \\
& +\operatorname{Pr}\left(q_{n}\left(X^{n}, Y^{n}\right) \geq \tau_{n}^{*}+2 \epsilon\right) .
\end{aligned}
$$

Now, since $P^{(n)}$ is uniform in a single type-class, $q_{n}\left(X^{n}, Y^{n}\right)-\mathbb{E}_{P^{(n)} \times W^{(n)}}\left(q_{n}\right)$ converges in probability to 0 (see 1163), Claim 11). Hence, by definition of $\tau_{n}^{*}$, both $\operatorname{Pr}\left(\left\{q_{n}\left(X^{n}, Y^{n}\right)<\tau_{n}^{*}\right\}\right)$ and $\operatorname{Pr}\left(q_{n}\left(X^{n}, Y^{n}\right) \geq \tau_{n}^{*}+2 \epsilon\right)$ tend to zero as $n$ tends to infinity. Consequently from [113, (115), for all $\delta>0$ there exists $n(\delta)$ such that for all $n \geq n(\delta)$,

$$
\frac{1}{2} \operatorname{Pr}\left\{M_{n} \Phi_{q_{n}}\left(\tau_{n}^{*}, P^{(n)}, Y^{n}\right)>1\right\} \leq \operatorname{Pr}\left(\exists j \neq i: q_{n}\left(X^{n}(j), Y^{n}\right) \geq q_{n}\left(X^{n}, Y^{n}\right)-2 \epsilon\right)+\delta,
$$

Now, the term $\operatorname{Pr}\left(\exists j \neq i: q_{n}\left(X^{n}(j), Y^{n}\right) \geq q_{n}\left(X^{n}, Y^{n}\right)-2 \epsilon\right)$ is the ensemble average probability of error using the mismatched decoder metric $q^{\prime}(x, y)=q(x, y)-2 \epsilon$. Since $q_{n}\left(X^{n}, Y^{n}\right)$ converges in probability to $\mathbb{E}_{P^{(n)} \times W^{(n)}}\left(q_{n}\right)$, and the empirical distribution of $Y^{n}, \hat{P}_{Y^{n}}$, converges in probability to $P_{Y}$, for $\Delta>0$ arbitrarily small, with probability approaching $1, q_{n}\left(X^{n}, Y^{n}\right) \geq \mathbb{E}_{P W}(q)-\Delta$ and there exists a vanishing sequence $\zeta_{n}$ such that

$$
\begin{aligned}
& \operatorname{Pr}\left(\exists j \neq i: q_{n}\left(X^{n}(j), Y^{n}\right) \geq q_{n}\left(X^{n}, Y^{n}\right)-2 \epsilon\right) \\
\leq & M_{n} \cdot(n+1)^{|\mathcal{X}|(|\mathcal{Y}|+1)} 2^{-n \min _{\tilde{P}_{X, Y}: \mathbb{E}_{\tilde{P}}(q) \geq \mathbb{E}_{P W}(q)-2 \epsilon-\Delta, \tilde{P}_{X}=P_{X}, \tilde{P}_{Y}=P_{Y}} I_{\tilde{P}}(X ; Y)}+\zeta_{n} \\
= & 2^{n\left(R-C_{q-2 \epsilon-\Delta}^{(1)}(W)+|\mathcal{X}|(|\mathcal{Y}|+1) \frac{\log (n+1)}{n}\right)}+\zeta_{n},
\end{aligned}
$$


where the inequality follows from the union bound and because the number of joint type-classes over $\mathcal{X} \times \mathcal{Y}$ is upper bounded by $(n+1)^{|\mathcal{X}||\mathcal{Y}|-1}$, and $C_{q}^{(1)}(W)$ is defined in (97). In light of (116) and (117), if for some $\epsilon_{n}>0$ one has $R<C_{q+2 \epsilon-\Delta}^{(1)}(W)-|\mathcal{Y}|(|\mathcal{X}|+1) \frac{\log (n+1)}{n}$ the term $\operatorname{Pr}\left\{M_{n} \Phi_{q_{n}}\left(\tau_{n}^{*}, P^{(n)}, Y^{n}\right)>1\right\}$ must vanish, and thus the r.h.s. of (112) is upper bounded by $C_{q-2 \epsilon-\Delta}^{(1)}(W)$. In other words, by continuity of $C_{q}^{(1)}(W)$ in $q$, the threshold decoder achieves a rate arbitrarily close to $C_{q}^{(1)}(W)$. The same argument holds for $C_{q}^{(K)}(W), K>1$ (with slower rate of convergence), and thus, since $\lim _{K \rightarrow \infty} C_{q}^{(K)}(W)=C_{q}^{(\infty)}(W)$ the threshold decoder achieves a rate arbitrarily close to $C_{q}^{(\infty)}(W)$ for $n$ sufficiently large.

We note that for a similar argument that was used in (111), the right hand side of 1112 ) is equal to

$$
\sup _{\boldsymbol{P}: \forall n, P^{(n)} \in \mathcal{P}_{C C}(\mathcal{X}, n)} \sup _{\epsilon>0} p-\liminf -\frac{1}{n} \log \left(\Phi_{q_{n}}\left(\mathbb{E}_{\hat{P}_{X^{n}} \times W}\left(q_{n}(X, Y)\right)-\epsilon, P^{(n)}, Y^{n}\right)\right) .
$$

Theorem 8 reinforces Csiszár and Narayan's conjecture and thus solves Open Problem 6 in [1, Section 5] as well as two additional problems:

- Open Problem 5 [1, Section 5]: It is stated that $C_{q}(W)=C_{q}^{(\infty)}(W)$ is a sufficient condition for the existence of rate approaching $R<C_{q}(W)$ codes and probability of error decaying to zero exponentially as the block length goes to infinity, and due to Theorem 8 , this is indeed the case.

- Open Problem 7 of [1] concerns the threshold $q$-capacity, which, as states in [1], is clearly upper bounded by the mismatch $q$-capacity (see Lemma 3 for the proof in the general channel case). Further, $C_{q}^{(\infty)}(W)$ constitutes a lower bound on the threshold $q$-capacity too ( [2], [3]). Hence, as states in [1], the affirmative answer to Problem 6 implies that the constant threshold capacity of the DMC is equal to the mismatch capacity. This claim is also proved in Lemma 6 (see (112)).

As mentioned before, Theorem 8 was proved in [1] for the special case of erasures-only capacity of the DMC. We noted that the erasures only capacity can be considered as the supremum of rates achievable by decoding with respect to the metric $q_{n}^{e o}$ (see (90)). In the DMC case, this metric is equivalent (capacity-wise) to the additive metric

$$
q_{n, e o}\left(x^{n}, y^{n}\right)=\sum_{i=1}^{n} 1\left\{W\left(y_{i} \mid x_{i}\right)>0\right\},
$$

i.e., with $q(x, y)=1\{W(y \mid x)>0\}$, and similarly to the metric $q_{n}^{e o}$, in this special case, it always holds that the actual transmitted codeword $X^{n}$ and received signal $Y^{n}$ satisfy

$$
q_{n, e o}\left(X^{n}, Y^{n}\right)=1
$$

Hence, the erasures-only capacity $C_{e o}(W)$ is equal to the threshold capacity with threshold levels $\tau=1$ (see (72)), and Lemma 6 applied to this metric in the DMC case yields an alternative expression to the erasures-only capacity

\footnotetext{
${ }^{5}$ In this case, one need not take a threshold level $\tau$ arbitrarily close to 1 but rather set $\tau=1$ since the equality 119 holds surely, for every realization of $\left(X^{n}, Y^{n}\right)$.
} 
of the DMC $W, C_{e o}(W)$, which is given by

$$
\begin{aligned}
C_{q_{e o}}^{(\infty)}(W) & =\sup _{\boldsymbol{P} \in \mathcal{P}(\infty)} p-\liminf -\frac{1}{n} \log \left(\Phi_{q_{n, e o}}\left(1, P^{(n)}, Y^{n}\right)\right) \\
& =\sup _{\boldsymbol{P} \in \mathcal{P}(\infty)} p-\liminf -\frac{1}{n} \log \left(\sum_{\tilde{x}^{n}: W^{n}\left(Y^{n} \mid \tilde{x}^{n}\right)>0} P^{(n)}\left(\tilde{x}^{n}\right)\right),
\end{aligned}
$$

where the supremum can be restricted to $\left\{\boldsymbol{P} \in \mathcal{P}_{U}^{(\infty)}\right\}$.

We note that using Corollary 1 (see (28) and (31) we obtain the bound

$$
C_{q_{e o}}^{(\infty)}(W) \leq \sup _{\boldsymbol{P} \in \mathcal{P}_{U}^{(\infty)}} \liminf _{n \rightarrow \infty} \frac{1}{n} \mathbb{E}\left(\log \frac{1}{\sum_{\tilde{x}^{n}: W^{n}\left(Y^{n} \mid \tilde{x}^{n}\right)>0} P^{(n)}\left(\tilde{x}^{n}\right)}\right),
$$

which was recently established in [24, Theorem 2.1], and as pointed out in [24], it is easy to show that the right hand side of 122) with the liminf replaced by limsup is an achievable rate as the $n$-letter extension of Forney's [25] lower bound on $C_{e o}(W)$, and therefore the above inequality holds with equality, i.e.,

$$
C_{q_{e o}}^{(\infty)}(W)=\sup _{\boldsymbol{P} \in \mathcal{P}_{U}^{(\infty)}} \lim _{n \rightarrow \infty} \frac{1}{n} \mathbb{E}\left(\log \frac{1}{\sum_{\tilde{x}^{n}: W^{n}\left(Y^{n} \mid \tilde{x}^{n}\right)>0} P^{(n)}\left(\tilde{x}^{n}\right)}\right) .
$$

\section{RAndom Coding Over a Given Codebook}

In this section, we establish a connection between the maximal probability of erroneously decoding a message using a codebook $\mathcal{C}_{n}$ and a decoder $q_{n}$ and the average probability of the random codebook, of slightly lower rate, whose codewords are drawn i.i.d. over $\mathcal{C}_{n}$ using the same decoder. This derivation, beyond being interesting in itself, enables to establish an alternative proof of Theorem 11, which is based on the analysis of the performance of a random code. Along with the direct part of the proof of Theorem 1 it constitutes a complete proof which is based on random coding.

Recall that $P_{e}\left(W^{(n)}, \mathcal{C}_{n}, q_{n}\right)$ denotes the average probability of error incurred using the $\left(M_{n}, n\right)$-code $\mathcal{C}_{n}$ with the decoder $q_{n}$ (4) where $\boldsymbol{y}$ is the output of the channel $W^{(n)}$. Let

$$
P_{\max }\left(W^{(n)}, \mathcal{C}_{n}, q_{n}\right)=\max _{i \in\left\{1, \ldots, M_{n}\right\}} \operatorname{Pr}(\hat{S} \neq S \mid S=i)
$$

be the maximal probability of error incurred by the same code and decoder.

Denote by $\bar{P}_{e}\left(W^{(n)}, \mathcal{C}_{n}, q_{n}, R-\epsilon\right)$ the average probability of error of the random code of size

$$
M_{n, \epsilon}=2^{n(R-\epsilon)}
$$

with independent codewords, each drawn uniformly over $\mathcal{C}_{n}$ using the same decoder $q_{n}$.

We next present a lemma which implies that $\bar{P}_{e}\left(W^{(n)}, \mathcal{C}_{n}, q_{n}, R-\epsilon\right)$ can be upper bounded by $P_{\max }\left(W^{(n)}, \mathcal{C}_{n}, q_{n}\right)$ up to a vanishing quantity.

Lemma 7. If $\mathcal{C}_{n}$ is a codebook composed of $M_{n}=2^{n R}$ distinct codewords and $\epsilon \in(0, R)$, then

$$
\bar{P}_{e}\left(W^{(n)}, \mathcal{C}_{n}, q_{n}, R-\epsilon\right) \leq P_{\max }\left(W^{(n)}, \mathcal{C}_{n}, q_{n}\right)+\delta_{n}
$$


where

$$
\delta_{n} \triangleq \frac{1}{2} \cdot\left(2^{-n \epsilon}-2^{-n R}\right)
$$

Proof: Let $\mathcal{C}_{n}=\left\{\boldsymbol{x}_{1}, \ldots, \boldsymbol{x}_{M_{n}}\right\}$ be the given codebook. Let $q_{n}$ be a decoder of the form (4). Next draw $M_{n, \epsilon}=2^{n(R-\epsilon)}$ codewords independently using $P^{(n)} \in \mathcal{P}\left(\mathcal{X}^{n}\right)$, and assign indices to the drawn codewords by their order of appearance. The codewords constitute the random codebook $\tilde{\mathcal{C}}_{n}=\left\{\tilde{\boldsymbol{X}}_{1}, \ldots, \tilde{\boldsymbol{X}}_{M_{n, \epsilon}}\right\}$. Let $\mathcal{B}$ be the random set of codewords which appear only once in $\tilde{\mathcal{C}}_{n}$, let $X^{n}$ be the transmitted random codeword (uniformly distributed over $\tilde{\mathcal{C}}_{n}$ ), and let $S$ and $\hat{S}$ stand for the transmitted message and the output of the decoder $q_{n}$, respectively.

One has

$$
\begin{aligned}
& \bar{P}_{e}\left(W^{(n)}, \mathcal{C}_{n}, q_{n}, R-\epsilon\right) \\
= & \operatorname{Pr}\left(\hat{S} \neq S, X^{n} \in \mathcal{B}\right)+\operatorname{Pr}\left(\hat{S} \neq S, X^{n} \in \mathcal{B}^{c}\right) \\
= & \operatorname{Pr}\left(X^{n} \in \mathcal{B}\right) \operatorname{Pr}\left(\hat{S} \neq S \mid X^{n} \in \mathcal{B}\right)+\operatorname{Pr}\left(X^{n} \in \mathcal{B}^{c}\right) \operatorname{Pr}\left(\hat{S} \neq S \mid X^{n} \in \mathcal{B}^{c}\right) \\
\stackrel{(a)}{=} & \left(1-1 / M_{n}\right)^{M_{n, \epsilon}-1} \cdot \operatorname{Pr}\left(\hat{S} \neq S \mid X^{n} \in \mathcal{B}\right)+\left[1-\left(1-1 / M_{n}\right)^{M_{n, \epsilon}-1}\right] \cdot \operatorname{Pr}\left(\hat{S} \neq S \mid X^{n} \in \mathcal{B}^{c}\right) \\
\leq & \operatorname{Pr}\left(\hat{S} \neq S \mid X^{n} \in \mathcal{B}\right)+\left[1-\left(1-1 / M_{n}\right)^{M_{n, \epsilon}-1}\right] \cdot \operatorname{Pr}\left(\hat{S} \neq S \mid X^{n} \in \mathcal{B}^{c}\right)
\end{aligned}
$$

where $(a)$ follows since $\operatorname{Pr}\left(X^{n} \in \mathcal{B}\right)=\sum_{\boldsymbol{x} \in \mathcal{C}} \frac{1}{M_{n}} \operatorname{Pr}\left(\cap_{i=2}^{M_{n, \epsilon}}\left\{\tilde{\boldsymbol{X}}_{i} \neq \boldsymbol{x}\right\}\right)=\sum{\boldsymbol{x} \in \mathcal{C}_{n}}_{\frac{1}{M}} \prod_{i=2}^{M_{n, \epsilon}}\left(1-1 / M_{n}\right)$.

Now, note that

$$
\begin{aligned}
& 1-\left(1-1 / M_{n}\right)^{M_{n, \epsilon}-1} \\
\geq & \frac{1}{2} \min \left\{1,\left(M_{n, \epsilon}-1\right) / M_{n}\right\} \\
= & \frac{1}{2} \cdot\left(2^{-n \epsilon}-2^{-n R}\right),
\end{aligned}
$$

where the inequality follows from (114). Therefore, we obtain

$$
\begin{aligned}
& \bar{P}_{e}\left(W^{(n)}, \mathcal{C}_{n}, q_{n}, R-\epsilon\right) \\
\leq & \operatorname{Pr}\left(\hat{S} \neq S \mid X^{n} \in \mathcal{B}\right)+\delta_{n} \cdot \operatorname{Pr}\left(\hat{S} \neq S \mid X^{n} \in \mathcal{B}^{c}\right) \\
& \stackrel{(a)}{\leq} P_{\max }\left(W^{(n)}, \mathcal{C}_{n}, q_{n}\right)+\delta_{n}
\end{aligned}
$$

where (a) follows because given $X^{n} \in \mathcal{B}$, it is known that $X^{n}$ appears only once in the codebook, while other codewords may appear more than once, and using the decoder $q_{n}$ of the form (4) with a subset of the codewords of the original codebook enlarges the decision regions and cannot increase the maximal probability of error.

Having proved Lemma 7 we can establish an alternative proof of the converse part of Theorem 1 
Proof: As a result of Lemma 7, to obtain an upper bound on the capacity (w.r.t. maximal probability of error), we can analyze the average probability of error of the average code whose codewords are drawn uniformly over a codebook $\mathcal{C}_{n}$. Let $P^{(n)} \in \mathcal{P}\left(\mathcal{X}^{n}\right)$ be uniform over $\mathcal{C}_{n}$. Clearly, for sufficiently large $n$

$$
\begin{aligned}
& P_{\max }\left(W^{(n)}, \mathcal{C}_{n}, q_{n}\right)+\delta_{n} \\
\stackrel{(a)}{\geq} & \bar{P}_{e}\left(W^{(n)}, \mathcal{C}_{n}, q_{n}, R-\epsilon\right) \\
\stackrel{(b)}{=} & \mathbb{E}\left[1-\left(1-\Phi_{q_{n}}\left(q_{n}\left(X^{n}, Y^{n}\right), P^{(n)}, Y^{n}\right)\right)^{\left(M_{n, \epsilon}-1\right)}\right] \\
\geq & \mathbb{E}\left(\left[1-\left(1-\Phi_{q_{n}}\left(q_{n}\left(X^{n}, Y^{n}\right), P^{(n)}, Y^{n}\right)\right)^{\left(M_{n, \epsilon}-1\right)}\right] 1\left\{\left(M_{n, \epsilon}-1\right) \cdot \Phi_{q_{n}}\left(q_{n}\left(X^{n}, Y^{n}\right), P^{(n)}, Y^{n}\right) \geq e^{n \gamma}\right\}\right) \\
\geq & \mathbb{E}\left(\left[1-\left(1-\frac{e^{n \gamma}}{M_{n, \epsilon}-1}\right)^{\left(M_{n, \epsilon}-1\right)}\right] 1\left\{\left(M_{n, \epsilon}-1\right) \cdot \Phi_{q_{n}}\left(q_{n}\left(X^{n}, Y^{n}\right), P^{(n)}, Y^{n}\right) \geq e^{n \gamma}\right\}\right) \\
\stackrel{(c)}{\geq} & {\left[1-e^{\left.-e^{n \gamma}\right]} \mathbb{E}\left(1\left\{\left(M_{n, \epsilon}-1\right) \cdot \Phi_{q_{n}}\left(q_{n}\left(X^{n}, Y^{n}\right), P^{(n)}, Y^{n}\right) \geq e^{n \gamma}\right\}\right)\right.} \\
\geq & \operatorname{Pr}\left\{\left(M_{n, \epsilon}-1\right) \Phi_{q_{n}}\left(q_{n}\left(X^{n}, Y^{n}\right), P^{(n)}, Y^{n}\right) \geq e^{n \gamma}\right\}-e^{-e^{n \gamma}},
\end{aligned}
$$

where ( $a$ ) follows from Lemma7 (b) follows since the decoder successfully decodes $S=m$ only if $q_{n}(\boldsymbol{x}(m), \boldsymbol{y})>$ $q_{n}(\boldsymbol{x}(j), \boldsymbol{y})$ for all $j \neq m$ and an error occurs if there is at least one "failure" in $M_{n, \epsilon}-1$ Bernoulli experiments, and $(c)$ follows from the inequality $\left(1-L^{-1}\right)^{L} \leq e^{-1}$ applied to $L=\frac{M_{n, \epsilon}-1}{e^{n \gamma}}$.

Next, for all $\delta>0$, there exists sufficiently large $n$ such that, $M_{n, \epsilon}-1 \geq M_{n, \epsilon} 2^{-n \delta}=M 2^{-n(\epsilon+\delta)}$, thus for sufficiently large $n$,

$$
\begin{aligned}
& \operatorname{Pr}\left\{\left(M_{n, \epsilon}-1\right) \Phi_{q_{n}}\left(q_{n}\left(X^{n}, Y^{n}\right), P^{(n)}, Y^{n}\right) \geq e^{n \gamma}\right\} \\
= & \operatorname{Pr}\left\{-\frac{1}{n} \log \left(\Phi_{q_{n}}\left(q_{n}\left(X^{n}, Y^{n}\right), P^{(n)}, Y^{n}\right)\right) \leq \frac{1}{n} \log \left(M_{n, \epsilon}-1\right)-\gamma\right\} \\
\geq & \operatorname{Pr}\left\{-\frac{1}{n} \log \left(\Phi_{q_{n}}\left(q_{n}\left(X^{n}, Y^{n}\right), P^{(n)}, Y^{n}\right)\right) \leq \frac{1}{n} \log \left(M_{n}\right)-\epsilon-\delta-\gamma\right\} .
\end{aligned}
$$

To conclude, we have for sufficiently large $n$, a weaker version of 197 :

$$
\begin{aligned}
& P_{\max }\left(W^{(n)}, \mathcal{C}_{n}, q_{n}\right)+\delta_{n} \\
\geq & \operatorname{Pr}\left\{-\frac{1}{n} \log \left(\Phi_{q_{n}}\left(q_{n}\left(X^{n}, Y^{n}\right), P^{(n)}, Y^{n}\right)\right) \leq \frac{1}{n} \log (M)-\epsilon-\delta-\gamma\right\}-e^{-e^{n \gamma} .}
\end{aligned}
$$

It is easy to realize that since $\delta_{n} \rightarrow 0$ as $n$ tends to infinity, by definition of the limit inferior in probability, if

$$
R>\sup _{\boldsymbol{P}} p-\liminf -\frac{1}{n} \log \left(\Phi_{q_{n}}\left(q_{n}\left(X^{n}, Y^{n}\right), P^{(n)}, Y^{n}\right)\right)=\underline{K}_{\boldsymbol{q}}(\boldsymbol{W}),
$$

the maximal probability of error does not vanish as $n$ tends to infinity. This part of the proof follows similarly to equations (3.11)-(3.14) in [20] as follows: We show that the assumption that $R=R_{0}=\underline{K}_{\boldsymbol{q}}(\boldsymbol{W})+3 \gamma+\delta+\epsilon$ is achievable leads to a contradiction for arbitrarily small positive $\gamma, \epsilon, \delta$. Since by assumption $R_{0}$ is achievable, for all $\zeta>0$ and $n$ sufficiently large, there exists an $\left(n, M_{n}, \epsilon_{n}\right)$-code satisfying

$$
\liminf _{n \rightarrow \infty} \frac{1}{n} \log M_{n} \geq R_{0}-\zeta
$$


and $\lim _{n \rightarrow \infty} \epsilon_{n}=0$. Define $X^{n}$ as the random variable uniformly distributed over the code, and $Y^{n}$ the corresponding output, we then have from (133) for all $\gamma>0$ and sufficiently large $n$,

$$
\operatorname{Pr}\left\{-\frac{1}{n} \log \left(\Phi_{q_{n}}\left(q_{n}\left(X^{n}, Y^{n}\right), P^{(n)}, Y^{n}\right)\right) \leq \frac{1}{n} \log M_{n}-\epsilon-\delta-\gamma\right\} \leq \epsilon_{n}+\delta_{n}+e^{-e^{n \gamma}} .
$$

On the other hand from for $n$ sufficiently large, it holds that

$$
\operatorname{Pr}\left\{-\frac{1}{n} \log \left(\Phi_{q_{n}}\left(q_{n}\left(X^{n}, Y^{n}\right), P^{(n)}, Y^{n}\right)\right) \leq R_{0}-\epsilon-\delta-\gamma-\zeta\right\} \leq \epsilon_{n}+\delta_{n}+e^{-e^{n \gamma}} .
$$

Now, pick $\zeta=\gamma$ and substitute $R_{0}=\underline{K}_{\boldsymbol{q}}(\boldsymbol{W})+3 \gamma+\epsilon+\delta$, this yields

$$
\operatorname{Pr}\left\{-\frac{1}{n} \log \left(\Phi_{q_{n}}\left(q_{n}\left(X^{n}, Y^{n}\right), P^{(n)}, Y^{n}\right)\right) \leq \underline{K}_{\boldsymbol{q}}(\boldsymbol{W})+\gamma\right\} \leq \epsilon_{n}+\delta_{n}+e^{-e^{n \gamma}} .
$$

However, the definition of liminf in probability implies the existence of $\epsilon>0$ such that for infinitely many $n$ 's satisfying

$$
\operatorname{Pr}\left\{-\frac{1}{n} \log \left(\Phi_{q_{n}}\left(q_{n}\left(X^{n}, Y^{n}\right), P^{(n)}, Y^{n}\right)\right) \leq \underline{K}_{\boldsymbol{q}}(\boldsymbol{W})+\gamma\right\} \geq \epsilon,
$$

since $\lim _{n \rightarrow \infty} \epsilon_{n}=0$ this yields a contradiction.

Finally, by observing that the mismatch capacity w.r.t. maximal probability of error is equal to the mismatch capacity w.r.t. average probability of error, this concludes the second proof of the converse part of Theorem 1.

\section{Conditions For the Existence of A Strong CONVERse}

In this section, we present a condition which is necessary and sufficient for a channel with mismatched decoding to have a strong converse. We say that a channel satisfies the strong converse property if for all $\delta>0$, every sequence of $\left(M_{n}, n, \epsilon_{n}\right)$-codes with $\frac{1}{n} \log M_{n}>C_{\boldsymbol{q}}(\boldsymbol{W})+\delta, \forall n$, satisfies $\lim _{n \rightarrow \infty} \epsilon_{n}=1$. Recall the definition of $\bar{K}_{\boldsymbol{q}}(\boldsymbol{W})$ (44).

Theorem 9. A channel $\boldsymbol{W}$ satisfies the strong converse iff

$$
\bar{K}_{\boldsymbol{q}}(\boldsymbol{W})=\underline{K}_{\boldsymbol{q}}(\boldsymbol{W})
$$

The necessary and sufficient condition for the existence of a strong converse which was established by Verdú and Han [20] in the matched metric case is

$$
\sup _{\boldsymbol{P}} \bar{I}\left(X^{n} ; Y^{n}\right)=\sup _{\boldsymbol{P}} \underline{I}\left(X^{n} ; Y^{n}\right)
$$

and thus, 140 for $q=W$ is equivalent to (141). This is not surprising in light of (48).

Proof: Sufficiency: To prove sufficiency of condition [140), we assume a sequence of codes $\left\{\mathcal{C}_{n}\right\}_{n \geq 1}$ is given such that

$$
\frac{1}{n} \log \left(M_{n}\right) \geq \underline{K} \boldsymbol{q}(\boldsymbol{W})+\delta
$$


Let $P^{(n)}$ be the uniform distribution over $\mathcal{C}_{n}$, and $\left(X^{n}, Y^{n}\right) \sim P^{(n)} \times W^{(n)}$. Now, from Lemma 1 we have

$$
\begin{aligned}
& P_{e}\left(W^{(n)}, \mathcal{C}_{n}, q_{n}\right) \\
= & \operatorname{Pr}\left\{-\frac{1}{n} \log \left(\Phi_{q_{n}}\left(q_{n}\left(X^{n}, Y^{n}\right), P^{(n)}, Y^{n}\right)\right)<\frac{1}{n} \log M_{n}\right\} \\
\geq & \operatorname{Pr}\left\{-\frac{1}{n} \log \left(\Phi_{q_{n}}\left(q_{n}\left(X^{n}, Y^{n}\right), P^{(n)}, Y^{n}\right)\right)<\underline{K}_{\boldsymbol{q}}(\boldsymbol{W})+\delta\right\} \\
= & \operatorname{Pr}\left\{-\frac{1}{n} \log \left(\Phi_{q_{n}}\left(q_{n}\left(X^{n}, Y^{n}\right), P^{(n)}, Y^{n}\right)\right)<\bar{K}_{\boldsymbol{q}}(\boldsymbol{W})+\delta\right\}
\end{aligned}
$$

where the last step follows from (140). By definition of the limit superior in probability and by the fact that we take the supremum over $\boldsymbol{P}$ in the definition of $\bar{K}_{\boldsymbol{q}}(\boldsymbol{W})$, the r.h.s. of (143) must go to 1 as $n$ tends to infinity, and hence so must the 1.h.s. and the channel has a strong converse.

Necessity: To prove necessity of condition [140), we assume that the channel satisfies the strong converse property. Let $\mathcal{G}\left(n, M_{n}\right)$ denote the set of $\left(n, M_{n}\right)$-codebooks over $\mathcal{X}^{n}$. Denote $\left.M_{n}^{\Delta} \triangleq\left\lceil 2^{n(\underline{K}} \boldsymbol{q}(\boldsymbol{W})+\Delta\right)\right\rceil$. Thus, for all $\Delta>0$,

$$
\liminf _{n \rightarrow \infty} \inf _{\mathcal{C}_{n} \in \cup_{M_{n} \geq M_{n}^{\Delta}}} P_{\mathcal{G}} P_{e}\left(W^{(n)}, \mathcal{C}_{n}, q_{n}\right)=1 .
$$

Clearly, one can assume that the infimum is attained within $\mathcal{G}\left(n, M_{n}^{\Delta}\right)$ without loss of generality, since for every $\left(n, M_{n}\right)$-codebook $\mathcal{C}_{n}$ and every $M_{n}^{\prime}<M_{n}$, there exists a sub-codebook $\mathcal{C}_{n}^{\prime}$ of size $M_{n}^{\prime}$ such that $P_{e}\left(W^{(n)}, \mathcal{C}_{n}^{\prime}, q_{n}\right) \leq P_{e}\left(W^{(n)}, \mathcal{C}_{n}, q_{n}\right)$. Hence, for all $\Delta>0$

$$
\liminf _{n \rightarrow \infty} \inf _{\mathcal{C}_{n} \in \mathcal{G}\left(n, M_{n}^{\Delta}\right)} P_{e}\left(W^{(n)}, \mathcal{C}_{n}, q_{n}\right)=1
$$

Now, since a nearly optimal code $\mathcal{C}_{n}^{*}$ (i.e., such that $P_{e}\left(W^{(n)}, \mathcal{C}_{n}^{*}, q_{n}\right) \leq \inf _{\mathcal{C}_{n} \in \mathcal{G}\left(n, M_{n}^{\Delta}\right)} P_{e}\left(W^{(n)}, \mathcal{C}_{n}, q_{n}\right)+\epsilon$ for arbitrarily small $\epsilon>0$ ) performs at least as well, in terms of average probability of error, as any code ensemble, i.e.,

$$
\begin{aligned}
& \inf _{\mathcal{C}_{n} \in \mathcal{G}\left(n, M_{n}^{\Delta}\right)} P_{e}\left(W^{(n)}, \mathcal{C}_{n}, q_{n}\right) \\
\leq & \inf _{\mu \in \mathcal{P}\left(\left(\mathcal{X}^{n}\right)^{M_{n}^{\Delta}}\right)} \int d \mu\left(\mathcal{C}_{n}\right) P_{e}\left(W^{(n)}, \mathcal{C}_{n}, q_{n}\right)
\end{aligned}
$$

and since the code ensemble which is drawn with independent codewords each drawn according to some $P^{(n)} \in$ $\mathcal{P}\left(\mathcal{X}^{n}\right)$ is a special case of a random code, we have

$$
\begin{gathered}
\inf _{\mu \in \mathcal{P}\left(\left(\mathcal{X}^{n}\right)^{M_{n}^{\Delta}}\right)} \int d \mu\left(\mathcal{C}_{n}\right) P_{e}\left(W^{(n)}, \mathcal{C}_{n}, q_{n}\right) \\
\leq \inf _{P^{(n)}} \mathbb{E}\left[1-\left(1-\Phi_{q_{n}}\left(q_{n}\left(X^{n}, Y^{n}\right), P^{(n)}, Y^{n}\right)\right)^{M_{n}^{\Delta}-1}\right]
\end{gathered}
$$


thus, we obtain

$$
\begin{aligned}
& \inf _{\mathcal{C}_{n} \in \mathcal{G}\left(n, M_{n}^{\Delta}\right)} P_{e}\left(W^{(n)}, \mathcal{C}_{n}, q_{n}\right) \\
& \leq \inf _{P^{(n)}} \mathbb{E}\left[1-\left(1-\Phi_{q_{n}}\left(q_{n}\left(X^{n}, Y^{n}\right), P^{(n)}, Y^{n}\right)\right)^{M_{n}^{\Delta}-1}\right] \\
& \stackrel{(a)}{\leq} \inf _{P^{(n)}} \mathbb{E} \min \left\{1, M_{n}^{\Delta} \Phi_{q_{n}}\left(q_{n}\left(X^{n}, Y^{n}\right), P^{(n)}, Y^{n}\right)\right\}
\end{aligned}
$$

where $(a)$ follows from the union bound. Now, let $\boldsymbol{P}=\left\{P^{(i)}\right\}_{i \geq 1}$ be given, and let $\overline{\mathcal{A}}_{n}$ denote the set of pairs of $n$-vectors $(\boldsymbol{x}, \boldsymbol{y}) \in \mathcal{X}^{n} \times \mathcal{Y}^{n}$ such that $M_{n}^{\Delta} \cdot \Phi_{q_{n}}\left(q_{n}(\boldsymbol{x}, \boldsymbol{y}), P^{(n)}, \boldsymbol{y}\right) \leq 2^{-n \gamma}$ we have

$$
\begin{aligned}
& \mathbb{E} \min \left\{1, M_{n}^{\Delta} \Phi_{q_{n}}\left(q_{n}\left(X^{n}, Y^{n}\right), P^{(n)}, Y^{n}\right)\right\} \\
\leq & \mathbb{E}\left(1\left\{\left(X^{n}, Y^{n}\right) \in \overline{\mathcal{A}}_{n}\right\} \min \left\{1, M_{n}^{\Delta} \Phi_{q_{n}}\left(q_{n}\left(X^{n}, Y^{n}\right), P^{(n)}, Y^{n}\right)\right\}\right) \\
& +\operatorname{Pr}\left\{\overline{\mathcal{A}}_{n}^{c}\right\} \\
\leq & 2^{-n \gamma}+\operatorname{Pr}\left\{\overline{\mathcal{A}}_{n}^{c}\right\} \\
= & 2^{-n \gamma}+\operatorname{Pr}\left\{-\frac{1}{n} \log \left(\Phi_{q_{n}}\left(q_{n}\left(X^{n}, Y^{n}\right), P^{(n)}, Y^{n}\right)\right)<\underline{K}_{\boldsymbol{q}}(\boldsymbol{W})+\Delta+\delta_{n}+\gamma\right\},
\end{aligned}
$$

where $\delta_{n}$ is such that $\frac{1}{n} \log M_{n}^{\Delta}=\frac{1}{n} \log \left\lceil 2^{n(\underline{K} \boldsymbol{q}(\boldsymbol{W})+\Delta)}\right\rceil=2^{n\left(\underline{K} \boldsymbol{q}(\boldsymbol{W})+\Delta+\delta_{n}\right)}$, i.e., $\lim _{n \rightarrow \infty} \delta_{n}=0$. Summarizing 144-149 we obtain

$$
1 \leq \liminf _{n \rightarrow \infty} \inf _{P^{(n)}} \operatorname{Pr}\left\{-\frac{1}{n} \log \left(\Phi_{q_{n}}\left(q_{n}\left(X^{n}, Y^{n}\right), P^{(n)}, Y^{n}\right)\right)<\underline{K}_{\boldsymbol{q}}(\boldsymbol{W})+\Delta+\delta_{n}+\gamma\right\} .
$$

This yields

$$
\underline{K}_{\boldsymbol{q}}(\boldsymbol{W})+\Delta+\gamma \geq \bar{K}_{\boldsymbol{q}}(\boldsymbol{W})
$$

and since $\Delta+\gamma$ can be made arbitrarily small we have

$$
\underline{K}_{\boldsymbol{q}}(\boldsymbol{W}) \geq \bar{K}_{\boldsymbol{q}}(\boldsymbol{W})
$$

and along with the obvious opposite inequality, the equality (140) follows.

The next lemma extends Corollary 1 and shows that if the channel satisfies the strong converse property then (28) holds with equality.

Lemma 8. If the channel $\boldsymbol{W}$ has a finite input alphabet then

$$
\begin{aligned}
\underline{K}_{\boldsymbol{q}}(\boldsymbol{W}) & \leq \liminf _{n \rightarrow \infty} \sup _{P^{(n)}} \frac{1}{n} \mathbb{E} \log \frac{1}{\Phi_{q_{n}}\left(q_{n}\left(X^{n}, Y^{n}\right), P^{(n)}, Y^{n}\right)} \\
& \leq \limsup _{n \rightarrow \infty} \sup _{P^{(n)}} \frac{1}{n} \mathbb{E} \log \frac{1}{\Phi_{q_{n}}\left(q_{n}\left(X^{n}, Y^{n}\right), P^{(n)}, Y^{n}\right)} \leq \bar{K} \boldsymbol{q}(\boldsymbol{W}),
\end{aligned}
$$

and if in addition, the channel satisfies the strong converse property,

$$
C_{\boldsymbol{q}}(\boldsymbol{W})=\lim _{n \rightarrow \infty} \sup _{P^{(n)}} \frac{1}{n} \mathbb{E} \log \frac{1}{\Phi_{q_{n}}\left(q_{n}\left(X^{n}, Y^{n}\right), P^{(n)}, Y^{n}\right)},
$$

where the supremums in (153)-(154) can be restricted to $P^{(n)}$ that is uniform over a subset of $\mathcal{X}^{n}$. 
Proof: The leftmost inequality (153) was established in (32), and in fact, it holds for more general alphabets. It remains to prove the rightmost inequality assuming the alphabet $\mathcal{X}^{n}$ is finite. The proof follows similarly to the proof of [21, Theorem 3.5.2.]. Let $\left\{A_{n}\right\}_{n \geq 1}$ be a sequence of non-negative random variables, and denote $\bar{A} \triangleq p-\lim \sup A_{n}$. If $\bar{A}<c<\infty$, then one has for all $\epsilon>0$,

$$
\begin{aligned}
\mathbb{E}\left(A_{n}\right) & =\mathbb{E}\left(A_{n} 1\left\{A_{n} \geq c\right\}\right)+\mathbb{E}\left(A_{n} 1\left\{c>A_{n} \geq \bar{A}+\epsilon\right\}\right)+\mathbb{E}\left(A_{n} 1\left\{A_{n}<\bar{A}+\epsilon\right\}\right) \\
& \leq \mathbb{E}\left(A_{n} 1\left\{A_{n} \geq c\right\}\right)+c \cdot \mathbb{E}\left(1\left\{A_{n}>\bar{A}+\epsilon\right\}\right)+(\bar{A}+\epsilon) \mathbb{E}\left(1\left\{A_{n}<\bar{A}+\epsilon\right\}\right),
\end{aligned}
$$

by definition of $\bar{A}$, and since $\epsilon$ can be made arbitrarily small, this yields that

$$
\limsup _{n \rightarrow \infty} \mathbb{E}\left(A_{n}\right) \leq \bar{A}+\limsup _{n \rightarrow \infty} \mathbb{E}\left(A_{n} 1\left\{A_{n} \geq c\right\}\right) .
$$

Now, observe that $-\frac{1}{n} \log \Phi_{q_{n}}\left(q_{n}\left(X^{n}, Y^{n}\right), P^{(n)}, Y^{n}\right) \leq-\frac{1}{n} \log P^{(n)}\left(X^{n}\right) \triangleq Z_{n}$ and take $c=|\mathcal{X}|+\epsilon$ (which is an upper bound on $p$ - $\limsup -\frac{1}{n} \log P^{(n)}\left(X^{n}\right)$ [21, Theorem 1.7.2.]). It was proved in [21, Theorem 3.5.2.] that

$$
\limsup _{n \rightarrow \infty} \mathbb{E}\left(Z_{n} 1\left\{Z_{n} \geq|\mathcal{X}|+\epsilon\right\}\right)=0,
$$

and thus, the rightmost inequality of (153) follows. The equality (154) follows from Theorem 9

\section{CONCLUSION}

This paper presents a derivation of a general formula for the mismatch $\boldsymbol{q}$-capacity, $C_{\boldsymbol{q}}(\boldsymbol{W})$, of the channel $\boldsymbol{W}$. The general capacity formula is given in terms of the supremum over input distributions sequence of the limit inferior in probability of the exponent of the conditional error probability given the channel input and output in a single drawing of another codeword uniformly over the codebook. We provide two proofs for the upper bound on $C_{\boldsymbol{q}}(\boldsymbol{W})$ : The first proof is based on an extension of the Verdú-Han upper bound for the general channel capacity formula. The second proof is based on lower bounding (up to a vanishing quantity) the average error probability of a rate- $R$ codebook $\mathcal{C}$ by the average error probability of the ensemble random code of rate $R-\epsilon$ whose codewords are drawn independently over $\mathcal{C}$.

Comparing the general capacity formula applied to the matched metric and the Verdú-Han channel capacity formula yields an interesting identity between the supremum over input distribution sequence of the limit inferior in probability of the two sequences of random variables (a) $\frac{1}{n} \log \frac{W^{(n)}\left(Y^{n} \mid X^{n}\right)}{P_{Y}\left(Y^{n}\right)}$ - the mutual information density rate of $\left(X^{n}, Y^{n}\right)$, the channel input and output, and (b) $-\frac{1}{n} \log \left(\Phi_{W^{(n)}}\left(W^{(n)}\left(Y^{n} \mid X^{n}\right), P^{(n)}, Y^{n}\right)\right)$ - the exponent of the conditional error probability given $\left(X^{n}, Y^{n}\right)$ in a single drawing of another codeword $\tilde{X}^{n}$ uniformly over the codebook in dependently of $\left(X^{n}, Y^{n}\right)$.

Using the insight gained from the derivation of the general capacity formula, we derive two max-min upper bounds on the capacity in terms of supremum over input processes of the infimum over a class of channels of the resulting spectral inf-mutual information rates. A lower bound on the mismatch capacity of the channel $W$ with a non-negative decoding metric is derived, which is tight in the matched case. We further provide necessary and 
sufficient conditions for a channel to have a strong converse. We study the closely related problem of threshold mismatched decoding, and obtain a general expression for the threshold mismatch capacity and the constant threshold mismatch capacity. The erasures-only general capacity formula is established as a special case.

Another contribution of this paper is a proof of the Csiszár and Narayan's conjecture [1, Open Problem 6], i.e., that the product space improvement of the random coding lower bound, $C_{q}^{(\infty)}(W)$, is indeed the mismatch capacity of the DMC $W$ with bounded additive decoding metric. We conclude by proving that in the DMC case, the constant threshold mismatch capacity is equal to the mismatch capacity and by deriving an identity between the two expressions.

\section{APPENDIX}

\section{A. Proof of Theorem 8}

As mentioned before, the fact that $C_{q}^{(\infty)}(W)$ is an achievable rate was pointed out in [1] as a generalization of the random coding bound $C_{q}^{(1)}(W)$ for the product channel $W^{K}$ with input and output alphabets $\mathcal{X}^{K}$ and $\mathcal{Y}^{K}$, respectively. The proof of the upper bound is divided into 9 steps:

Step 1: First, observe that without loss of asymptotic optimality, one can assume that the codebook contains codewords that lie in a single type-class. To be more precise, for any given $\left(n, 2^{n R}, \epsilon_{n}\right)$-code, one can find an $\left(n, 2^{n R^{\prime}}, 2 \epsilon_{n}\right)$ sub-code, where

$$
R^{\prime}=R-\frac{(|\mathcal{X}|-1) \log (2(n+1))}{n},
$$

whose codewords lie in a single type-class. To realize this, first expurgate half of the codewords that have the highest probability of error. This results in a codebook whose maximal error probability is upper bounded by $2 \epsilon_{n}$. Now, pick the dominant type-class (in the sense that its intersection with the remaining codewords is the largest), this leaves at least $1 /(n+1)^{(|\mathcal{X}|-1)}$ of the codewords with maximal (and thus also average) probability of error upper bounded by $2 \epsilon_{n}$.

Step 2: Next, it is easily verified, using the union bound, that if $\mathcal{C}_{n}$ is an $\left(n, 2^{n R^{\prime}}, 2 \epsilon_{n}\right)$-code, then applying $\mathcal{C}_{n}$ repeatedly $K_{n}=o\left(\epsilon_{n}^{-1}\right)$ times over the DMC, say

$$
K_{n}=\epsilon_{n}^{-1 / 2}
$$

times, results in average probability of error not exceeding $K_{n} \times 2 \epsilon_{n}=2 \epsilon_{n}^{1 / 2}$. For convenience denote

$$
N \triangleq n K_{n}
$$

Thus, without loss of asymptotic optimality, we can replace the codebook $\mathcal{C}_{n}$ with the codebook $\mathcal{C}_{N}^{\text {prod }}$ which is a $K_{n}$-times concatenation of the code $\mathcal{C}_{n}$. In other words, the message $m \in\left\{1, \ldots, 2^{N R^{\prime}}\right\}$ is split to $K_{n}$ sub-messages, $\left(m_{1}, \ldots, m_{K_{n}}\right)$, such that $m_{i} \in\left\{1, \ldots, 2^{n R^{\prime}}\right\}, \forall i$, and each message is mapped to $\mathcal{X}^{n}$ using the original codebook $\mathcal{C}_{n}$. Note that $\mathcal{C}_{N}^{\text {prod }}$ and $\mathcal{C}_{n}$ share the same rate.

From Steps 1 and 2 it follows that without loss of asymptotic optimality, we can assume that the codebook is a concatenation of $K_{n}$ uses of an $\left(n, 2^{n R^{\prime}}, 2 \epsilon_{n}\right)$-code whose codewords lie in a single type-class. 
Step 3: Note that by construction, also all the codewords of the codebook $\mathcal{C}_{N}^{\text {prod }}$ lie in a single type-class of sequences of length $N$.

Step 4: Now, let $X^{N}$ be the output of the encoder which uses the codebook $\mathcal{C}_{N}^{\text {prod }}$ and let $Y^{N}$ be the output of the channel $W^{N}$ when fed by $X^{N}$. We next show that the condition (52) is always satisfied for a memoryless channel $W^{(N)}=W^{N}$ with $\tau_{n}=\mathbb{E}_{P^{(N)} \times W^{(N)}}\left(q_{N}\right)+\Delta$ for all $\Delta>0$ and vanishingly small $\zeta_{N}=\epsilon_{2, N}$, i.e.,

$$
\operatorname{Pr}\left\{q_{N}\left(X^{N}, Y^{N}\right) \geq \mathbb{E}_{P^{(N)} \times W^{(N)}}\left(q_{N}\right)+\Delta\right\} \leq \epsilon_{2, N},
$$

where

$$
\epsilon_{2, N} \triangleq(n+1)^{|\mathcal{X}||\mathcal{Y}|} \exp \left\{-n \min _{\hat{P}_{y^{N} \mid x^{N}}:\left|\mathbb{E}_{\hat{P}_{x^{N}}, y^{N}}(q)-\mathbb{E}_{\hat{P}_{x^{N}} \times W}(q)\right| \geq \Delta} D\left(\hat{P}_{y^{N} \mid x^{N}} \| W \mid \hat{P}_{x^{N}}\right)\right\} .
$$

The following claim states this straightforward argument. Recall the definition of $\mathcal{P}_{C C}(\mathcal{X}, N)$ (1).

Claim 1. If $P^{(N)} \in \mathcal{P}_{C C}(\mathcal{X}, N)$ then

$$
\operatorname{Pr}\left\{\left|q_{N}\left(X^{N}, Y^{N}\right)-\mathbb{E}\left(q_{N}\left(X^{N}, Y^{N}\right)\right)\right| \geq \Delta\right\} \leq \epsilon_{2, N},
$$

where $\epsilon_{2, N}$ is defined in (162).

Proof: To prove the claim, pick an arbitrary $x^{N}$ that lies in the type-class over which $P^{(N)}$ is defined, note that by symmetry, $\mathbb{E}_{P^{(N)} \times W^{(N)}}\left(q_{N}\right)=\mathbb{E}\left(q_{N}\left(X^{N}, Y^{N}\right)\right)=\mathbb{E}\left(q_{N}\left(X^{N}, Y^{N}\right) \mid X^{N}=x^{N}\right)=\mathbb{E}_{\hat{P}_{x^{N}} \times W}(q)$ and thus,

$$
\begin{aligned}
& \operatorname{Pr}\left\{\left|q_{N}\left(X^{N}, Y^{N}\right)-\mathbb{E}\left(q_{N}\left(X^{N}, Y^{N}\right)\right)\right| \geq \Delta\right\} \\
= & \operatorname{Pr}\left\{\left|q_{N}\left(X^{N}, Y^{N}\right)-\mathbb{E}_{\hat{P}_{x^{N}} \times W}(q)\right| \geq \Delta\right\} \\
= & \operatorname{Pr}\left\{\left|q_{N}\left(x^{N}, Y^{N}\right)-\mathbb{E}_{\hat{P}_{x^{N}} \times W}(q)\right| \geq \Delta \mid X^{n}=x^{N}\right\} \\
= & \quad \sum_{\hat{P}_{y^{N} \mid x^{N}}:\left|\mathbb{E}_{\hat{P}_{x^{N}, y^{N}}}(q)-\mathbb{E}_{\hat{P}_{x^{N}} \times W}(q)\right| \geq \Delta} \operatorname{Pr}\left\{T\left(\hat{P}_{y^{N} \mid x^{N}}\right) \mid X^{n}=x^{N}\right\} \\
\leq & \epsilon_{2, N} \rightarrow 0 .
\end{aligned}
$$

Step 5: From Steps 1-3 we know that $P_{e}\left(W^{N}, \mathcal{C}_{N}^{\text {prod }}, q_{N}\right) \leq 2 \epsilon_{n}^{1 / 2}$. Let $\tilde{W}^{(N)}$ be a channel which satisfies

$$
\begin{aligned}
& \operatorname{Pr}\left\{q_{N}\left(X^{N}, \tilde{Y}^{(N)}\right) \leq \mathbb{E}\left(q_{N}\left(X^{N}, Y^{N}\right)\right)+\Delta\right\} \leq \epsilon_{1, N} \\
& P_{Y^{N}}=P_{\tilde{Y}^{N}},
\end{aligned}
$$

for some $\Delta>0$ and $\epsilon_{1, N}>0$, where $\tilde{Y}^{N}$ is the output of $\tilde{W}^{(N)}$ when fed by $X^{N}$, which is uniformly distributed over $\mathcal{C}_{N}^{\text {prod }}$. Note that (161), (165), (166) are in fact the conditions (52)-(54) required for Theorem 3 to hold, and hence

$$
\begin{aligned}
P_{e}\left(\tilde{W}^{(N)}, \mathcal{C}_{N}^{\text {prod }}, q_{N}\right) & \leq P_{e}\left(W^{N}, \mathcal{C}_{N}^{\text {prod }}, q_{N}\right)+\epsilon_{1, N}+\epsilon_{2, N} \\
& \leq 2 \epsilon_{n}^{1 / 2}+\epsilon_{1, N}+\epsilon_{2, N} \triangleq \bar{\epsilon}_{N} .
\end{aligned}
$$


Step 6: Denote the following set of block memoryless distributions

$$
\mathcal{P}_{N}^{\text {prod }} \triangleq\left\{P^{(N)} \in \mathcal{P}\left(\mathcal{X}^{N}\right): P^{(N)}=\prod_{k=0}^{K_{n}-1} \boldsymbol{P}^{(k)}, \forall k \boldsymbol{P}^{(k)} \in \mathcal{P}_{C C}(\mathcal{X}, n)\right\},
$$

where $\mathcal{P}_{C C}(\mathcal{X}, n)$ is defined in (1).

Now, from the Steps 1-5, we can invoke Fano's Inequality for the channel $\tilde{W}^{(n)}$ and this yields

$$
\begin{aligned}
& R-\frac{(|\mathcal{X}|-1) \log (2(n+1))}{n} \\
\leq & \max _{P^{(N)} \in \mathcal{P}_{N}^{\text {prod }} \tilde{W}^{(N)} \in \mathcal{W}_{b}\left(P^{(N)}, \epsilon_{N, 1}\right)} \frac{1}{N} I\left(X^{N} ; \tilde{Y}^{N}\right)+\frac{1}{N}+R \cdot \bar{\epsilon}_{N},
\end{aligned}
$$

where $\left(X^{N}, \tilde{Y}^{N}\right) \sim P^{(N)} \times \tilde{W}^{(N)}, \bar{\epsilon}_{N}$ is defined in 167) as a function of $\left(\epsilon_{N, 1}, \epsilon_{2, N}\right)$ with $\epsilon_{2, N}$ defined in (162), and where

$$
\mathcal{W}_{b}\left(P^{(n)}, \epsilon_{N, 1}\right) \triangleq\left\{\tilde{W}^{(N)}: \mathbb{E}_{P^{(N)} \times \tilde{W}^{(N)}}\left(1\left\{q_{N}\left(X^{N}, \tilde{Y}^{(N)}\right) \leq c_{N}+\Delta\right\}\right) \leq \epsilon_{N, 1}, P_{\tilde{Y}^{N}}=P_{Y^{N}}\right\},
$$

with

$$
c_{N} \triangleq \mathbb{E}_{P^{(N)} \times W^{(N)}}\left(q_{N}\left(X^{N}, Y^{N}\right)\right) .
$$

We note that in 169 ) we can take the minimum over $\tilde{W}^{(N)} \in \mathcal{W}_{b}\left(P^{(N)}, \epsilon_{N, 1}\right)$ rather than the infimum because $\mathcal{W}_{b}\left(P^{(N)}, \epsilon_{N, 1}\right)$ is a convex compact set.

Step 7: For $k=0,1, . ., K_{n}-1$ let

$$
\boldsymbol{X}^{(k)} \triangleq\left(X_{k n+1}, X_{k n+2}, \ldots, X_{(k+1) n}\right),
$$

i.e.,

$$
X^{N}=\left(\boldsymbol{X}_{(0)}, \ldots, \boldsymbol{X}_{\left(K_{n}-1\right)}\right)
$$

Denote

$$
c^{(k)} \triangleq \mathbb{E}_{P^{(N)} \times W^{(N)}}\left(q_{n}\left(\boldsymbol{X}^{(k)}, \boldsymbol{Y}^{(k)}\right)\right),
$$

and note that since the metric is additive we have

$$
c_{N}=\frac{1}{K_{n}} \sum_{k=0}^{K_{n}-1} c^{(k)}
$$

where $c_{N}$ is defined in (171).

Claim 2. Let $\Delta>0$ and $\delta>\Delta$ be arbitrarily small, and consider a block-wise memoryless channel $\tilde{W}^{(N)}=$ $\prod_{k=0}^{K_{n}-1} \tilde{\boldsymbol{W}}^{(k)}$ (where $\tilde{\boldsymbol{W}}^{(k)}$ is a channel from $\mathcal{X}^{n}$ to $\mathcal{Y}^{n}$ ) be given. If

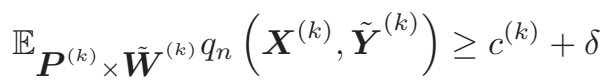

for all $k=1, \ldots, K_{n}$, then

$$
{ }_{\prod_{k=0}^{K_{n}-1} \boldsymbol{P}^{(k)} \times \tilde{\boldsymbol{W}}^{(k)}}^{\mathbb{E}}\left(1\left\{q_{N}\left(X^{N}, \tilde{Y}^{(N)}\right) \leq c_{N}+\Delta\right\}\right) \leq \epsilon_{1, N}
$$


where

$$
\epsilon_{1, N} \triangleq \frac{B^{2}}{K_{n}(\delta-\Delta)^{2}}
$$

and $B$ is the bound on $q$ (96).

Proof: The claim follows from the weak law of large numbers (LLN) for a sequence of independent random variables $\left\{A_{k}\right\}_{k=0}^{K_{n}-1}$ where

$$
A_{k}=q_{n}\left(\boldsymbol{X}^{(k)}, \tilde{\boldsymbol{Y}}^{(k)}\right)
$$

whose expectations are not necessarily identical. Recall the definition of $K_{n}$ (159). Note that we have from the fact that $q$ is bounded by $B(96$ and from (176)

$$
\begin{aligned}
& c^{(k)}+\delta \leq \mathbb{E}\left(A_{k}\right) \leq B<\infty \\
& \operatorname{Var}\left(A_{k}\right) \leq B^{2}<\infty \forall k
\end{aligned}
$$

and consequently, since $\delta>\Delta$, from Chebishev's Inequality we obtain

$$
\begin{aligned}
& \mathbb{M}_{\prod_{k=0}^{K_{n}-1} \boldsymbol{P}^{(k)} \times \tilde{\boldsymbol{W}}^{(k)}\left(1\left\{q_{N}\left(X^{N}, \tilde{Y}^{(N)}\right) \leq c_{N}+\Delta\right\}\right)} \\
= & \operatorname{Pr}\left(\frac{1}{K_{n}} \sum_{k=0}^{K_{n}-1}\left[A_{k}-c^{(k)}\right] \leq \Delta\right) \\
= & \operatorname{Pr}\left(\frac{1}{K_{n}} \sum_{k=0}^{K_{n}-1}\left[A_{k}-c^{(k)}-\delta\right] \leq \Delta-\delta\right) \\
\leq & \operatorname{Pr}\left(\frac{1}{K_{n}} \sum_{k=0}^{K_{n}-1}\left[A_{k}-\mathbb{E}\left(A_{k}\right)\right] \leq-(\delta-\Delta)\right) \\
\leq & \frac{\left[\mathbb{E}\left(\frac{1}{K_{n}} \sum_{k=0}^{K_{n}-1}\left[A_{k}-\mathbb{E}\left(A_{k}\right)\right]\right)^{2}\right]}{(\delta-\Delta)^{2}} \\
\leq & \frac{B^{2}}{K_{n}(\delta-\Delta)^{2}} \triangleq \epsilon_{1, N}
\end{aligned}
$$

which vanishes since $K_{n} \rightarrow \infty$.

Hence, from Claim 2 we can deduce that for $\delta>\Delta$,

$$
\begin{aligned}
& \mathcal{W}_{a}\left(\prod_{k=0}^{K_{n}-1} \boldsymbol{P}^{(k)}, \delta\right)
\end{aligned}
$$

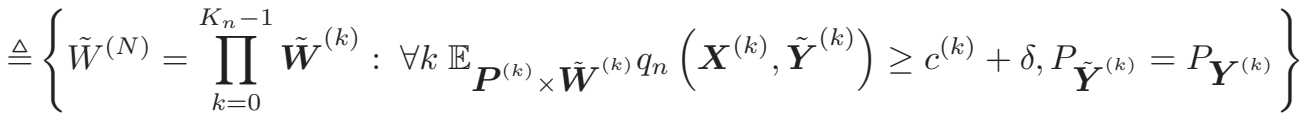

$$
\begin{aligned}
& \stackrel{(a)}{\subseteq}\left\{\tilde{W}^{(N)}=\prod_{k=0}^{K_{n}-1} \tilde{\boldsymbol{W}}^{(k)}: \begin{array}{l}
\prod_{k=0}^{K_{n}-1} \boldsymbol{P}^{(k)} \times \tilde{\boldsymbol{W}}^{(k)} \\
\forall k, P_{\tilde{\boldsymbol{Y}}^{(k)}}=P_{\boldsymbol{Y}^{(k)}}
\end{array}\right. \\
& \stackrel{(b)}{\subseteq} \mathcal{W}_{b}\left(\prod_{k=0}^{K_{n}-1} \boldsymbol{P}^{(k)}, \epsilon_{1, N}\right)
\end{aligned}
$$


where $\tilde{\boldsymbol{W}}^{(k)}$ is a channel from $\mathcal{Y}^{n}$ to $\mathcal{X}^{n},(a)$ follows from Claim 2 and $\epsilon_{1, N}$ is defined in (178), and (b) follows by definition of $\mathcal{W}_{b}\left(\prod_{k=0}^{K_{n}-1} \boldsymbol{P}^{(k)}, \epsilon_{1, N}\right)$ (see (170) $)$ and by the fact that the 1.h.s contains only block-wise memoryless channels.

We get from 181) and 159

$$
\epsilon_{1, N}=\frac{B^{2}}{K_{n}(\delta-\Delta)^{2}}=\frac{B^{2} \epsilon_{n}^{1 / 2}}{(\delta-\Delta)^{2}} .
$$

Step 8: Summarizing Steps 1-7, we obtain that for all $\Delta>0$ and $\delta>\Delta$, an achievable rate $R$ satisfies

$$
\begin{aligned}
R-\frac{(|\mathcal{X}|-1) \log (2(n+1))}{n} & \leq \max _{P^{(N)} \in \mathcal{P}_{N}^{\text {prod }}} \tilde{W}^{(N) \in \mathcal{W}_{b}\left(P^{(N)}, \epsilon_{N, 1}\right)} \\
& \left.\stackrel{(a)}{\leq} \max _{P^{(N)} \in \mathcal{P}_{N}^{\text {prod }}} \tilde{W}^{(N)} \min _{\left(\mathcal{W}_{a}\left(P^{(N)}, \delta\right)\right.} \frac{1}{N} I\left(X^{N} ; \tilde{Y}^{N}\right)+\frac{1}{N}+R \cdot \bar{\epsilon}_{N} ; \tilde{Y}^{N}\right)+\frac{1}{N}+R \cdot \bar{\epsilon}_{N}
\end{aligned}
$$

where $\bar{\epsilon}_{N}$ is defined in 1677, and (a) follows from [182, i.e., the fact that $\mathcal{W}_{a}\left(\prod_{k=0}^{K_{n}-1} \boldsymbol{P}^{(k)}, \delta\right) \subseteq$ $\mathcal{W}_{b}\left(\prod_{k=0}^{K_{n}-1} \boldsymbol{P}^{(k)}, \epsilon_{N, 1}\right)$.

Now, by definition of $\mathcal{W}_{a}\left(P^{(N)}, \delta\right), \tilde{W}^{(N)}$ is block-wise memoryless, and therefore

$$
\frac{1}{N} I\left(X^{N} ; \tilde{Y}^{N}\right) \leq \sum_{k=0}^{K_{n}-1} \frac{1}{N} I\left(\boldsymbol{X}^{(k)} ; \tilde{\boldsymbol{Y}}^{(k)}\right)
$$

Defining $\epsilon_{n}^{\prime} \triangleq \frac{1}{N}+R \cdot \bar{\epsilon}_{N}+\frac{(|\mathcal{X}|-1) \log (2(n+1))}{n}$ this yields

$$
\begin{aligned}
& R \leq \max _{P^{(N)} \in \mathcal{P}_{N}^{\text {prod }}} \min _{\tilde{W}^{(N)} \in \mathcal{W}_{a}\left(P^{(N)}, \delta\right)} \frac{1}{N} I\left(X^{N} ; \tilde{Y}^{N}\right)+\epsilon_{n}^{\prime} \\
& \leq \max _{P^{(N)} \in \mathcal{P}_{N}^{p r o d}} \min _{\tilde{W}^{(N)} \in \mathcal{W}_{a}\left(P^{(N)}, \delta\right)} \sum_{k=0}^{K_{n}-1} \frac{1}{N} I\left(\boldsymbol{X}^{(k)} ; \tilde{\boldsymbol{Y}}^{(k)}\right)+\epsilon_{n}^{\prime} \\
& =\max _{P^{(n)} \in \mathcal{P}_{C C}(\mathcal{X}, n) \tilde{W}^{(n)}: \mathbb{E}_{P^{(n)} \times \tilde{W}^{(n)}}\left(q_{n}\right) \geq \mathbb{E}_{P^{(n)} \times W^{(n)}}\left(q_{n}\right)+\delta, P_{Y^{n} n}=P_{Y^{n}}} \frac{1}{n} I_{P^{(n)} \times \tilde{W}^{(n)}}\left(X^{n} ; \tilde{Y}^{n}\right)+\epsilon_{n}^{\prime},
\end{aligned}
$$

where the last equality follows since the optimizations are in fact performed over block memoryless sources and channels.

Step 9: Next, we wish to take the limit of the right hand side of (186) as $\delta \rightarrow 0$ to obtain $C_{q}^{(\infty)}(W)$. Before taking the limit, we will treat the case in which the set over which the minimization is performed is empty.

To this aim, denote

$$
\begin{aligned}
\mathbb{E}_{P^{(N)} \times W^{N}}\left(q_{N}\left(X^{n}, Y^{n}\right)\right) & =\mathbb{E}_{\hat{P}_{X^{N}}} \max _{y \in \mathcal{Y}} q(X, y)-\delta_{1} \\
& \triangleq q_{\max }\left(\hat{P}_{X^{N}}\right)-\delta_{1}, .
\end{aligned}
$$

and note that since $P^{(N)} \in \mathcal{P}_{C C}(\mathcal{X}, N), q_{\max }\left(\hat{P}_{X^{N}}\right)$ is deterministic. In this case, since $P^{(N)} \in \mathcal{P}_{C C}(\mathcal{X}, N)$, we have

$$
\operatorname{Pr}\left\{q_{N}\left(X^{N}, Y^{N}\right)>q_{\max }\left(\hat{P}_{X^{N}}\right)\right\}=0 .
$$


Let $\tilde{W}^{(N)}$ be a channel which satisfies

$$
\begin{aligned}
& \operatorname{Pr}\left\{q_{N}\left(X^{N}, \tilde{Y}^{N}\right)<q_{\max }\left(\hat{P}_{X^{N}}\right)\right\}=0 \\
& P_{Y^{N}}=P_{\tilde{Y}^{N}} .
\end{aligned}
$$

We thus obtain the conditions (52)-(54) required for Theorem 3 to hold, with $n$ in lieu of $N, \tau_{N}=q_{\max }\left(\hat{P}_{X^{N}}\right)$, $\zeta_{N}=0, \eta_{N}=0$, and hence

$$
P_{e}\left(\tilde{W}^{(N)}, \mathcal{C}_{N}^{\text {prod }}, q_{N}\right) \leq P_{e}\left(W^{N}, \mathcal{C}_{N}^{\text {prod }}, q_{N}\right)
$$

Finally, by the additivity of the metric $q_{n}$, we have that if

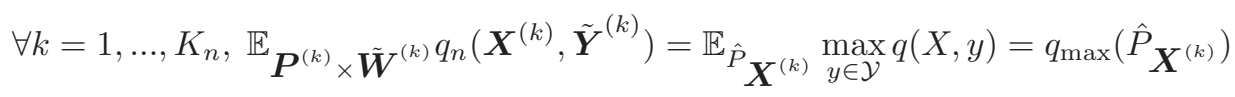

then

$$
\operatorname{Pr}\left\{q_{N}\left(X^{N}, \tilde{Y}^{N}\right)<q_{\max }\left(\hat{P}_{X^{N}}\right)\right\}=0,
$$

i.e., $q_{N}\left(X^{N}, \tilde{Y}^{N}\right)=q_{\max }\left(\hat{P}_{X^{N}}\right)$, and hence, we can repeat the derivation similarly to that of Step 8 by replacing $\mathcal{W}_{b}\left(P^{(N)}, \epsilon_{N, 1}\right)$ with the set of channels $\tilde{W}^{(N)}$ satisfying conditions $\left[189\right.$ ), and by replacing $\mathcal{W}_{a}\left(P^{(N)}, \delta\right)$ with the block-wise memoryless channels $\tilde{W}^{(N)}=\prod_{k=1}^{K_{n}} \tilde{\boldsymbol{W}}^{(k)}$ such that 191] holds. Summing up, we obtain

$$
R \leq \max _{P^{(n)} \in \mathcal{P}_{C C}(\mathcal{X}, n)} \min _{\tilde{W}^{(n)}: \mathbb{E}\left(q_{n}\left(X^{n}, \tilde{Y}^{n}\right)\right) \geq \mathbb{E}_{\hat{P}_{X^{n}}} \max _{y \in \mathcal{Y}} q(X, y), P_{Y^{n}}=P_{Y^{n}}} \frac{1}{n} I_{P^{(n)} \times \tilde{W}^{(n)}}\left(X^{n} ; \tilde{Y}^{n}\right)+\epsilon_{n}^{\prime} .
$$

Combined with (186) this yields

$$
\begin{aligned}
& R \leq \max _{P^{(n)} \in \mathcal{P}_{C C}(\mathcal{X}, n)} \\
& \left.\quad \tilde{W}^{(n): \mathbb{E}\left(q_{n}\left(X^{n}, \tilde{Y}^{n}\right)\right) \geq \min \left\{\mathbb{E}\left(q_{n}\left(X^{n}, Y^{n}\right)\right)+\delta, \mathbb{E}_{\hat{P}_{X^{n}}}\right.} \max _{y \in \mathcal{Y}} q(X, y)\right\}, P_{Y^{n}}=P_{Y^{n}} \\
& \frac{1}{n} I_{P^{(n)} \times \tilde{W}^{(n)}}\left(X^{n} ; \tilde{Y}^{n}\right)+\epsilon_{n}^{\prime},
\end{aligned}
$$

which concludes the proof of Step 9.

Finally, the above inequality holds for all $\delta>0$. Since the set over which the minimization is performed is convex and non empty, and $I_{P}(X ; Y), E_{P}(q)$ are continuous in $P$, taking the limit of the right hand side of (194) as $\delta \rightarrow 0$ yields $C_{q}^{(\infty)}(W)$ and Theorem 8 follows.

\section{REFERENCES}

[1] I. Csiszár and P. Narayan, "Channel capacity for a given decoding metric," IEEE Trans. Inf. Theory, vol. 41, no. 1, pp. 35-43, Jan. 1995.

[2] I. Csiszár and J. Körner, "Graph decomposition: A new key to coding theorems," IEEE Trans. Inf. Theory, vol. 27, no. 1, pp. 5-12, 1981.

[3] J. Hui, "Fundamental issues of multiple accessing," PhD dissertation, MIT, 1983.

[4] A. Lapidoth, "Mismatched decoding and the multiple-access channel," IEEE Trans. Inf. Theory, vol. 42, no. 5, pp. 1439-1452, Sept. 1996.

[5] A. Somekh-Baruch, "On achievable rates for channels with mismatched decoding," May 2013, arXiv 1305.0547 [cs.IT].

[6] A. Somekh-Baruch, "On coding schemes for channels with mismatched decoding," in Proc. Int. Symp. Information Theory, ISIT, Istanbul,Turkey, 2013.

[7] J. Scarlett, L. Peng, N. Merhav, A. Martinez, and A. Guillén i Fàbregas, "Superposition codes for mismatched decoding," in Proc. Int. Symp. Information Theory, ISIT, Istanbul,Turkey, 2013, pp. 81-85. 
[8] V. Balakirsky, "Coding theorem for discrete memoryless channels with given decision rule," in Algebraic Coding, ser. Lecture Notes in Computer Science, G. Cohen, A. Lobstein, G. Zémor, and S. Litsyn, Eds. Springer Berlin Heidelberg, 1992, vol. 573, pp. 142-150. [Online]. Available: http://dx.doi.org/10.1007/BFb0034351

[9] G. Kaplan and S. Shamai, "Information rates and error exponents of compound channels with application to antipodal signaling in a fading environment," AEU. Archiv für Elektronik und Übertragungstechnik, vol. 47, no. 4, pp. 228-239, 1993.

[10] N. Merhav, G. Kaplan, A. Lapidoth, and S. Shamai (Shitz), "On information rates for mismatched decoders," IEEE Trans. Inf. Theory, vol. 40, no. 6, pp. 1953-1967, Nov. 1994.

[11] Y.-S. Liu and B. Hughes, "A new universal random coding bound for the multiple-access channel," IEEE Trans. Inf. Theory, vol. 42, no. 2, pp. 376-386, Match 1996.

[12] A. Lapidoth, "Nearest neighbor decoding for additive non-gaussian noise channels," IEEE Trans. Inf. Theory, vol. 42, no. 5, pp. 1520-1529, Sept. 1996.

[13] A. Ganti, A. Lapidoth, and I. Telatar, "Mismatched decoding revisited: general alphabets, channels with memory, and the wide-band limit," IEEE Trans. Inf. Theory, vol. 46, no. 7, pp. 2315-2328, Nov. 2000.

[14] S. Shamai and I. Sason, "Variations on the gallager bounds, connections, and applications," Information Theory, IEEE Transactions on, vol. 48, no. 12, pp. 3029-3051, 2002.

[15] J. Scarlett and A. Guillén i Fàbregas, "An achievable error exponent for the mismatched multiple-access channel," in 50th Annual Allerton Conference on Communication, Control, and Computing (Allerton), October 2012, pp. 1975-1982.

[16] J. Scarlett, A. Martinez, and A. Guillén i Fàbregas, "Mismatched decoding: finite-length bounds, error exponents and approximations," arXiv:1303.6166 [cs.IT], March 2013.

[17] — " "Ensemble-tight error exponents for mismatched decoders," in 50th Annual Allerton Conference on Communication, Control, and Computing (Allerton), 2012, pp. 1951-1958.

[18] _ _ "Expurgated random-coding ensembles: exponents, refinements and connections," July 2013, arXiv 1307.6679 [cs.IT].

[19] V. B. Balakirsky, "A converse coding theorem for mismatched decoding at the output of binary-input memoryless channels," IEEE Trans. Inf. Theory, vol. 41, no. 6, pp. 1889-1902, Nov. 1995.

[20] S. Verdú and T. S. Han, “A general formula for channel capacity,” IEEE Trans. Inf. Theory, vol. 40, no. 4, pp. 1147-1157, July 1994.

[21] T. S. Han, Information-spectrum methods in Information Theory. Berlin: Springer, 2003.

[22] T. R. Fischer, "Some remarks on the role of inaccuracy in Shannons theory of information transmission," in Transactions of the Eighth Prague Conference. Springer, 1978, pp. 211-226.

[23] A. Somekh-Baruch and N. Merhav, "Achievable error exponents for the private fingerprinting game," IEEE Trans. Inf. Theory, vol. 53, no. 5, pp. 1827-1838, May 2007.

[24] C. Bunte, A. Lapidoth, and A. Samorodintsky, "The zero-undetected-error capacity approaches the Sperner capacity," September 2013, arXiv 1309.4930 [cs.IT].

[25] J. Forney, G.D., "Exponential error bounds for erasure, list, and decision feedback schemes," Information Theory, IEEE Transactions on, vol. 14 , no. 2, pp. 206-220, 1968. 